\title{
Free fall in KvN mechanics and Einstein's principle of equivalence
}

\author{
Abhijit Sen \\ Novosibirsk State University, Novosibirsk 630 090, Russia \\ Shailesh Dhasmana \\ Novosibirsk State University, Novosibirsk 630 090, Russia \\ Zurab K. Silagadze \\ Budker Institute of Nuclear Physics and Novosibirsk State University, Novosibirsk 630 \\ 090, Russia
}

\begin{abstract}
The implementation of Einstein's principle of equivalence in Koopman-von Neumann $(\mathrm{KvN})$ mechanics is discussed. The implementation is very similar to the implementation of this principle in quantum mechanics. This is not surprising, because $\mathrm{KvN}$ mechanics provides a Hilbert space formulation of classical mechanics that is very similar to the quantum mechanical formalism.

Both in $\mathrm{KvN}$ mechanics and quantum mechanics, a propagator in a homogeneous gravitational field is simply related with a free propagator. As a result, the wave function in a homogeneous gravitational field in a freely falling reference frame differs from the free wave function only in phase.

Fisher information, which quantifies our ability to estimate mass from coordinate measurements, does not depend on the magnitude of the homogeneous gravitational field, and this fact constitutes the formulation of Einstein's principle of equivalence, which is valid both in quantum mechanics and $\mathrm{KvN}$ mechanics.
\end{abstract}

Email addresses: abhijit913@gmail.com (Abhijit Sen), dx.shailesh@gmail.com (Shailesh Dhasmana), Z.K.Silagadze@inp.nsk.su (Zurab K. Silagadze) 


\section{Introduction}

The equivalence principle is at the heart of Einstein's general relativity. Usually one distinguishes between weak and strong principles of equivalence [1]. According to the weak principle of equivalence, the local gravitational acceleration is independent of the composition and structure of the matter being accelerated. The universality of free fall, which follows from the principle of weak equivalence, can be verified experimentally, it has played an important role historically and still remains the subject of intense theoretical and experimental research [2, 3, 4].

Strong principle of equivalence asserts much more [2, 44]:

1. The weak principle of equivalence is valid for self-gravitating bodies as well as for test bodies.

2. The outcome of any local test experiment is independent of the velocity and of the orientation of the freely falling laboratory in which the experiment is performed.

3. The outcome of any local test experiment is independent of where and when it is performed.

In contrast to the weak principle of equivalence, the strong principle of equivalence is somewhat ambiguous like the correspondence principle between classical and quantum mechanics [5]. For example, the second facet of the strong principle of equivalence, the local Lorentz invariance, is heuristically useful for generalizing physical laws tentatively known in the absence of gravity to the case when gravity is present. However, when the second or higher derivatives occur in the physical law, an ambiguity arises since the corresponding covariant derivatives do not commute. This ambiguity is analogous to the operator ordering ambiguity in the quantum-classical correspondence [5].

Another possible source of confusion in the formulation of the strong principle of equivalence is the term "local". From a practical point of view, this, of course, means that the laboratory should be small enough and the duration of the experiment short enough to neglect the effects of spacetime curvature. However, whether one can neglect the effects of the spacetime curvature depends also on the sensitivity of the laboratory apparatus.

It is not surprising, therefore, that several non-equivalent formulations of the principle of equivalence appeared in the literature in order to make the

principle more accurate [6]. Moreover, there is no universal agreement on the 
meaning and implementation of the equivalence principle within a relativistic framework (see references cited in [7]). Einstein himself always insisted on the fundamental importance of this principle to the general theory of relativity. This insistence created a puzzle for philosophers and historians of science [8], because to maintain Einstein's view that an accelerated laboratory in the absence of gravity is strictly equivalent to a laboratory at rest in a gravitational field, and thus acceleration, like velocity, is a relative quantity, one should assume sufficiently small (in fact, infinitesimal) spacetime regions and limit attention to sufficiently elementary phenomena, because otherwise, due to the presence of curvature, one can detect the difference between the two situations [6].

It is expected that the weak principle of equivalence will be violated in quantum mechanics if it is understood as the independence of the dynamics of a test body on its mass in an external gravitational field. A simple argument showing that not all consequences of the equivalence principle in the classical domain are valid in the quantum domain is as follows [9, 10].

Imagine a bound quantum system of neutron and electron in which the gravitational interaction is responsible for the bound state formation. One can easily see after some calculations that energy eigenvalues depend on the electron mass $m$ (more precisely, are proportional to $\mathrm{m}^{3}$ ). Therefore, the frequency of transition from one energy eigenstate to another will also depend on $m$. These transition frequencies, in principle, can be experimentally observed, measured and used to find the mass of the electron. In contrast, in classical theory, the dynamics of a satellite orbiting a massive body does not depend on the mass of the satellite and, therefore, cannot be used to determine its value.

The crux of the problem, when we try to extend the weak principle of equivalence in quantum domain, lies in the fact that, in contrast to the Newton equation, when considering the behavior of a quantum particle in an external gravitational field, the mass of the particle does not drop from the corresponding Schrödinger equation.

However, the same thing happens with a free quantum particle too and this circumstance offers a way to understand and generalize Einstein's principle of equivalence in the quantum domain [11]. Einstein's principle of equivalence is the assertion that a state of rest in a homogeneous gravitational field is physically indistinguishable from a state of uniform acceleration in a gravity-free space. And in this form, the principle is valid in the quantum domain too, since it can be shown that the solution of the Schrödinger equa- 
tion for a particle in a homogeneous gravitational field $\vec{g}$, as viewed from the reference frame undergoing a constant acceleration $\vec{a}$, coincides to the free particle wave function up to a phase, if $\vec{a}=\vec{g}[11,12]$. That is, in quantum mechanics, like classical general relativity or Newtonian gravity, all effects of gravity are cancelled in a freely falling reference frame.

It was shown by Koopman [13] and von Neumann [14] long ago that classical mechanics can be formulated in the language of Hilbert spaces, in the way which resembles operator formulation of quantum mechanics. The purpose of the present article is to investigate free fall in the framework of Koopman-von Neuman $(\mathrm{KvN})$ mechanics and show that Einstein's principle of equivalence is implemented in $\mathrm{KvN}$ mechanics in much the same way as in ordinary quantum mechanics.

We tried to make the paper as self-contained as possible, providing the necessary pedagogical details where, in our opinion, they were needed. As a result, the article contains a significant amount of review material which is organized into several appendices.

\section{Einstein's principle of equivalence and quantum mechanics}

The Schrödinger equation in the inertial frame $S$ (where the gravitational potential is present) is given by

$$
i \hbar \frac{\partial \psi(x, t)}{\partial t}=-\frac{\hbar^{2}}{2 m} \frac{\partial^{2} \psi(x, t)}{\partial x^{2}}+m g x \psi(x, t)=\left(\frac{\hat{p}^{2}}{2 m}+m g \hat{x}\right) \psi(x, t),
$$

where $\hat{x}$ and $\hat{p}$ operators satisfy the canonical commutation relation $[\hat{x}, \hat{p}]=$ $i \hbar$. The formal solution of this Schrödinger equation has the form

$$
\psi(x, t)=e^{-\frac{i}{\hbar}\left(\frac{\hat{p}^{2}}{2 m}+m g \hat{x}\right) t} \psi(x, 0)
$$

where $\psi(x, 0)$ is the wave function of the initial state. The true solution of (1) then can be found by disentangling noncommutative operators in this formal solution (such operator methods were pioneered by Richard Feynman [15, 16]).

To disentangle the exponential operator in (21), one can use the "left oriented" version of the generalized Baker-Campbell-Hausdorff formula [17]

$$
e^{\lambda(\hat{A}+\hat{B})}=e^{\frac{\lambda^{3}}{3 !}(2[\hat{B},[\hat{A}, \hat{B}]]+[\hat{A},[\hat{A}, \hat{B}]])} e^{\frac{\lambda^{2}}{2 !}[\hat{A}, \hat{B}]} e^{\lambda \hat{B}} e^{\lambda \hat{A}}
$$


This second order formula is valid if

$[\hat{A},[\hat{A},[\hat{A}, \hat{B}]]]=[\hat{B},[\hat{B},[\hat{A}, \hat{B}]]]=[\hat{A},[\hat{B},[\hat{A}, \hat{B}]]]=[\hat{B},[\hat{A},[\hat{A}, \hat{B}]]]=0$.

In particular, if we take $\lambda=-\frac{i}{\hbar} t, \hat{A}=\frac{\hat{p}^{2}}{2 m}, \hat{B}=m g \hat{x}$, then

$$
[\hat{A}, \hat{B}]=-i g \hbar \hat{p}, \quad[\hat{A},[\hat{A}, \hat{B}]]=0, \quad[\hat{B},[\hat{A}, \hat{B}]]=m g^{2} \hbar^{2}
$$

and applying (3) to (2), we obtain

$$
\psi(x, t)=e^{\frac{i}{\hbar} \frac{m g^{2} t^{3}}{3}} e^{\frac{i}{\hbar} \frac{g t^{2}}{2} \hat{p}} e^{-\frac{i}{\hbar} m g \hat{x} t} e^{-\frac{i}{\hbar} \frac{\hat{p}^{2}}{2 m} t} \psi(x, 0) .
$$

It is clear that $e^{-\frac{i}{\hbar} \frac{\hat{p}^{2}}{2 m} t} \psi(x, 0)=\phi(x, t)$ gives the wave function of a free particle (that is, $\phi(x, t)$ is a solution of the Schrödinger equation with zero potential). On the other hand, $e^{\frac{i}{\hbar} a \hat{p}}$ is a spatial translation operator and for any arbitrary well behaved function $f(x)$, we have $e^{\frac{i}{\hbar} a \hat{p}} f(x)=f(x+a)$. Therefore

$$
e^{\frac{i}{\hbar} \frac{g t^{2}}{2} \hat{p}} e^{-\frac{i}{\hbar} m g \hat{x} t} \phi(x, t)=e^{-\frac{i}{\hbar} m g\left(x+\frac{g t^{2}}{2}\right) t} \phi\left(x+\frac{g t^{2}}{2}, t\right),
$$

and (5) takes the form

$$
\psi(x, t)=e^{-\frac{i m g t}{\hbar}\left(x+\frac{g t^{2}}{6}\right)} \phi\left(x+\frac{g t^{2}}{2}, t\right) .
$$

Expressing $x$ in terms of $x^{\prime}$ through (C.1), we see that the phase in the above exponential prefactor exactly equals, as expected, to $\Lambda / \hbar$, with $\Lambda$ specified in (C.2).

To better understand the physical meaning of ([6), let's make the change of variables (C.1), which, with a passive interpretation, corresponds to a transition to a freely falling reference frame, in the Schrödinger equation (1). Then the following picture emerges [12, 18, 19].

It follows from (C.1) that

$$
\frac{\partial x^{\prime}}{\partial x}=1, \quad \frac{\partial x^{\prime}}{\partial t}=g t=g t^{\prime}, \quad \frac{\partial t^{\prime}}{\partial x}=0, \quad \frac{\partial t^{\prime}}{\partial t}=1 .
$$

Therefore,

$$
\frac{\partial}{\partial t}=\frac{\partial t^{\prime}}{\partial t} \frac{\partial}{\partial t^{\prime}}+\frac{\partial x^{\prime}}{\partial t} \frac{\partial}{\partial x^{\prime}}=\frac{\partial}{\partial t^{\prime}}+g t^{\prime} \frac{\partial}{\partial x^{\prime}}, \quad \frac{\partial}{\partial x}=\frac{\partial t^{\prime}}{\partial x} \frac{\partial}{\partial t^{\prime}}+\frac{\partial x^{\prime}}{\partial x} \frac{\partial}{\partial x^{\prime}}=\frac{\partial}{\partial x^{\prime}}
$$


and the Schrödinger equation (10) in new coordinates takes the form

$$
i \hbar\left(\frac{\partial}{\partial t^{\prime}}+g t^{\prime} \frac{\partial}{\partial x^{\prime}}\right) \psi\left(x^{\prime}, t^{\prime}\right)=-\frac{\hbar^{2}}{2 m} \frac{\partial^{2} \psi\left(x^{\prime}, t^{\prime}\right)}{\partial x^{\prime 2}}+m g\left(x^{\prime}-\frac{1}{2} g t^{\prime 2}\right) \psi\left(x^{\prime}, t^{\prime}\right) .
$$

Let's try a solution in the form

$$
\psi\left(x^{\prime}, t^{\prime}\right)=e^{\frac{i}{\hbar} \Lambda\left(x^{\prime}, t^{\prime}\right)} \phi\left(x^{\prime}, t^{\prime}\right), \Lambda\left(x^{\prime}, t^{\prime}\right)=\frac{1}{3} m g^{2} t^{\prime 3}-m g x^{\prime} t^{\prime} .
$$

Note that $\Lambda\left(x^{\prime}, t^{\prime}\right)$ is precisely the function specified in (C.2). Substituting (9) into (8), we get after some straightforward calculation

$$
i \hbar \frac{\partial \phi\left(x^{\prime}, t^{\prime}\right)}{\partial t^{\prime}}=-\frac{\hbar^{2}}{2 m} \frac{\partial^{2} \phi\left(x^{\prime}, t^{\prime}\right)}{\partial x^{\prime 2}}
$$

Therefore $\phi\left(x^{\prime}, t^{\prime}\right)$ satisfies the Schrödinger equation for a free particle.

Now the physical meaning of (6) is clear: in a freely falling reference frame the wave function of a particle, not subject to non-gravitational forces, differs from the wave function of a free particle only by a phase factor. This circumstance can be considered as a quantum-mechanical embodiment of Einstein's principle of equivalence.

However, there is a subtlety. Although the overall phase of the wave function is unobservable, the relative phase between its two components does have observable consequences, and the phase in (6) depends on the position of the particle. Due to this position dependence, if a beam of quantum particles is coherently split into two parts in a gravitational field and then recombined, the interference effects may appear that will be mass dependent [9].

A beautiful experiment of this type was performed by Colella, Overhauser and Werner [20] in which the quantum-mechanical phase shift of neutrons induced by Earth's gravitational field was observed.

In this experiment, an almost monoenergetic horizontal beam of thermal neutrons was coherently divided into two secondary beams using Bragg reflection in the first silicon crystalline wafer. The secondary beams move at different heights in the Earth's gravitational field, and then each again coherently splits in the second silicon slab. Two of the resulting beams are directed to the same point in the third silicon plate, where they overlap and interfere with each other. Finally, the outgoing interfering beams are detected by neutron counters. 
Assuming for simplicity the constant height difference $H$ between the interfering beams on the horizontal sections of their trajectories, the equation (6) tells us that, when the beams recombine, the accumulated phase difference will be $\Delta \Phi=m g t H / \hbar$. But $t=L / v$, where $L$ is the length of the parallelogram along which the beams travel, and $v=p / m=h / m \lambda$ is the velocity of the neutron with the de de Broglie wavelength $\lambda$. Therefore, the expected phase difference is [18]

$$
\Delta \Phi=\frac{m^{2} g \lambda}{2 \pi \hbar^{2}} A, \quad A=H L .
$$

Of course, this simplified treatment, although it correctly conveys the main idea of the experiment, says nothing about the real difficulties that accompanied the implementation of the COW experiment, which required incredible experimental and engineering skills in the field of neutron scattering [21].

The fact that the phase shift (11) depends explicitly on the neutron mass triggered a heated discussion with no consensus up to now on whether the principle of equivalence applies to quantum systems, and what form it should take in quantum domain [22]. For example, it was stated in the well-known textbook on quantum mechanics [23] that the COW experiment shows that gravity is not purely geometric at the quantum level, since the effect depends on $m / \hbar$.

In reality, however, the COW experiment simply shows that in nonrelativistic quantum mechanics gravity appears in the Schrödinger equation as any other force would. And this circumstance does not compromise the geometrical description of gravity. In fact, it is possible to obtain the phase shift (11) by considering the non-relativistic limit of the Dirac equation in the general relativistic Schwarzschild metric [24].

In classical mechanics, the weak equivalence principle has three complementary forms [25]: 1) free-fall trajectories do not depend on the masses of the test particles; 2) inertial and (passive) gravitational masses are equal; 3) it is always possible to locally eliminate the gravitational field by going into a free falling reference frame.

It is clear that the first form is problematic in quantum mechanics, since a quantum particle, in general, does not have a well-defined trajectory. A possible quantum generalization of this form of equivalence principle, namely that the wave function of a freely falling particle does not depend on its mass, is also not feasible, since the wave function does depend on the mass of the particle. 
However, this impossibility to give a quantum version of the first form of the equivalence principle does not mean, as it is sometimes claimed, that quantum mechanics violates the equivalence principle. The mass dependence of the quantum-mechanical wave function is completely natural, as the following reasoning shows [26].

Geodesic equation (B.14) (its relativistic version) follows from the variational principle $\delta S=0$, where $S=-m c \int d \tau$ is the covariant classical action. The resulting classical trajectory is independent of the mass $m$, which enters in the action just as a kinematic overall multiplying factor. However the actual value of $S$ along classical trajectory depends on $m$ (is proportional to it). This value of $S$ is classically irrelevant, since it is unobservable. Nonetheless, it is observable in quantum mechanics as the phase $S / \hbar$ of the wave function (in the semiclassical approximation). Although the values of the classical action on different neutron paths in the COW experiment differ by a very small amount $m g t H=m g H L / v$, this difference is not small compared to the Planck's constant and leads to the observed interference pattern [26].

The second and third versions of the equivalence principle retain their operational meaning in the quantum domain too. In fact, COW type experiments are quantum tests of the equality of inertial and (passive) gravitational masses, albeit with a modest accuracy of about 1\%. Despite many improvements to the original COW experiment, a long-standing small discrepancy of about $1 \%$ between theory and experiment remained. This discrepancy probably is related to the slight intrinsic misalignments between diffracting crystals [27].

To verify that the same phase shift as in the gravitational field $\vec{g}$ is induced without gravity, but instead having the acceleration $-\vec{g}$, Bonse and Wroblewski conducted an experiment with a horizontally oscillating interferometer [28] and measured the phase shift as a function of the maximum acceleration of the apparatus. The results confirmed Einstein's principle of equivalence in the quantum domain with an accuracy of about $4 \%$.

\section{Einstein's principle of equivalence and $\mathrm{KvN}$ mechanics}

According to Sudarshan, any KvN system can be considered as a subsystem of a genuine quantum system with doubled degrees of freedom and with a certain quantum dynamics. Therefore, it is expected that the implementation of the Einstein's principle of equivalence in $\mathrm{KvN}$ mechanics will 
be very similar to how it is done in quantum mechanics. This is indeed true as shown below.

The Schrödinger equation (D.29) for the potential $V(x)=m g x$ (a classical particle of mass $m$ in a uniform gravitational field $g$ in a negative $x$ direction) has the form

$$
i \hbar \frac{\partial \psi(x, X, t)}{\partial t}=\left(\frac{\hat{p} \hat{P}}{m}+m g \hat{X}\right) \psi(x, X, t) .
$$

Its formal solution is

$$
\psi(x, X, t)=e^{-\frac{i}{\hbar}\left(\frac{\hat{p} \hat{P}}{m}+m g \hat{X}\right) t} \psi(x, X, 0),
$$

where $\psi(x, X, 0)$ is the wave function of the initial state. Again we should disentangle noncommutative operators in this formal solution. For this purpose, we use the generalized Baker-Campbell-Hausdorff formula (3) with $\lambda=-\frac{i}{\hbar} t$, $\hat{A}=\frac{\hat{p} \hat{P}}{m}, \hat{B}=m g \hat{X}$. Then

$$
[\hat{A}, \hat{B}]=-i g \hbar \hat{P}, \quad[\hat{A},[\hat{A}, \hat{B}]]=0, \quad[\hat{B},[\hat{A}, \hat{B}]]=0,
$$

and applying (3) to (13) , we obtain

$$
\psi(x, X, t)=e^{\frac{i}{\hbar} \frac{g t^{2}}{2} \hat{P}} e^{-\frac{i}{\hbar} m g \hat{X} t} e^{-\frac{i}{\hbar} \frac{\hat{p} \hat{P}}{m} t} \psi(x, X, 0) .
$$

But $e^{-\frac{i}{\hbar} \frac{\hat{p} \hat{P}}{m} t} \psi(x, X, 0)=\phi(x, X, t)$ gives the wave function of a free particle $(\phi(x, X, t)$ is a solution of the Schrödinger equation (12) with $g=0)$. On the other hand, $e^{\frac{i}{\hbar} a \hat{P}}$ is a spatial translation operator in the $x$-direction, as the commutation relations (D.22) do indicate. Therefore

$$
e^{\frac{i}{\hbar} \frac{g t^{2}}{2} \hat{P}} e^{-\frac{i}{\hbar} m g \hat{X} t} \phi(x, X, t)=e^{-\frac{i}{\hbar} m g X t} \phi\left(x+\frac{g t^{2}}{2}, X, t\right),
$$

and (15) takes the form

$$
\psi(x, X, t)=e^{-\frac{i}{\hbar} m g X t} \phi\left(x+\frac{g t^{2}}{2}, X, t\right) .
$$

Now we obtain this result in a different way that also parallels the quantum-mechanical case, as considered in [18]. The following passive transformation

$$
t^{\prime}=t \quad x^{\prime}=x+\frac{g t^{2}}{2} \quad X^{\prime}=X
$$


describes the transition to a freely falling reference frame, the origin of which moves in the negative $x$-direction with acceleration $g$. In the new coordinates, the Schrödinger equation (12) takes the form

$$
i \hbar\left(\frac{\partial \psi}{\partial t^{\prime}}+g t^{\prime} \frac{\partial \psi}{\partial x^{\prime}}\right)=-\frac{\hbar^{2}}{m} \frac{\partial^{2} \psi}{\partial x^{\prime} \partial X^{\prime}}+m g X^{\prime} \psi .
$$

Let's try the solution in the form

$$
\psi\left(x^{\prime}, X^{\prime}, t^{\prime}\right)=\Phi\left(x^{\prime}, X^{\prime}, t^{\prime}\right) \exp \left[i \eta\left(x^{\prime}, X^{\prime}, t^{\prime}\right],\right.
$$

where $\Phi\left(x^{\prime}, X^{\prime}, t^{\prime}\right)$ satisfies the free Schrödinger equation

$$
i \hbar \frac{\partial \Phi}{\partial t^{\prime}}=-\frac{\hbar^{2}}{m} \frac{\partial^{2} \Phi}{\partial x^{\prime} \partial X^{\prime}}
$$

Substituting (19) into (12) and using (20), we get

$$
\begin{aligned}
& i \hbar\left[i \frac{\partial \eta}{\partial t^{\prime}} \Phi+g t^{\prime}\left(\frac{\partial \Phi}{\partial x^{\prime}}+i \frac{\partial \eta}{\partial x^{\prime}} \Phi\right)\right]= \\
& -\frac{\hbar^{2}}{m}\left[i \frac{\partial \Phi}{\partial x^{\prime}} \frac{\partial \eta}{\partial X^{\prime}}+i \frac{\partial^{2} \eta}{\partial x^{\prime} \partial X^{\prime}} \Phi+i \frac{\partial \eta}{\partial x^{\prime}} \frac{\partial \Phi}{\partial X^{\prime}}-\frac{\partial \eta}{\partial x^{\prime}} \frac{\partial \eta}{\partial X^{\prime}} \Phi\right]+m g X^{\prime} \Phi .
\end{aligned}
$$

The coefficients of $\Phi, \frac{\partial \Phi}{\partial x^{\prime}}$ and $\frac{\partial \Phi}{\partial X^{\prime}}$ must match separately. Since we have $\frac{\partial \Phi}{\partial X^{\prime}}$ only in one place, hence $\frac{\partial \eta}{\partial x^{\prime}}=0$. Therefore, unlike the quantum case, the phase does not depend on the configuration space coordinate $x^{\prime}$, but may depend on the hidden coordinate $X^{\prime}$. Then, matching coefficients of $\Phi$ and $\frac{\partial \Phi}{\partial x^{\prime}}$, we find

$$
-\hbar \frac{\partial \eta}{\partial t^{\prime}}=m g X^{\prime}
$$

and

$$
i \hbar g t^{\prime}=-i \frac{\hbar^{2}}{m} \frac{\partial \eta}{\partial X^{\prime}}
$$

Eq.(22) can be integrated with the result

$$
\eta=-\frac{m g t^{\prime}}{\hbar} X^{\prime}+h\left(X^{\prime}\right)
$$

where $h$ is an arbitrary function which must be a constant in order (23) to be valid. Therefore, up to this irrelevant constant in phase,

$$
\psi\left(x^{\prime}, X^{\prime}, t^{\prime}\right)=\Phi\left(x^{\prime}, X^{\prime}, t^{\prime}\right) \exp \left[-\frac{i}{\hbar} m g t^{\prime} X^{\prime}\right]
$$


which is equivalent to (16). Therefore, as in the quantum case, in a freely falling reference frame the wave function of a particle not subject to nongravitational forces differs from the wave function of a free particle only by a phase factor. However, in contrast to quantum mechanics, this phase depends only on a hidden coordinate, and not on the observable position of the particle, and thus cannot lead to any observable interference effects.

Still another parallel with quantum-mechanical considerations is provided by the following observation. Let us make the following canonical transformation:

$$
\begin{array}{ll}
\hat{x}=\frac{1}{\sqrt{2}}\left(\hat{x}_{1}-\hat{x}_{2}\right), \quad \hat{X}=\frac{1}{\sqrt{2}}\left(\hat{x}_{1}+\hat{x}_{2}\right), \\
\hat{p}=\frac{1}{\sqrt{2}}\left(\hat{p}_{1}+\hat{p}_{2}\right), \quad \hat{P}=\frac{1}{\sqrt{2}}\left(\hat{p}_{1}-\hat{p}_{2}\right) .
\end{array}
$$

In new coordinates the Hamiltonian $\hat{H}=m g \hat{X}+\frac{\hat{p} \hat{P}}{m}$ takes the form

$$
\hat{H}=\left(\frac{\hat{p}_{1}^{2}}{2 m}+m \frac{g}{\sqrt{2}} \hat{x}_{1}\right)-\left(\frac{\hat{p}_{2}^{2}}{2 m}+m \frac{(-g)}{\sqrt{2}} \hat{x}_{2}\right)=\hat{H}_{1}-\hat{H}_{2} .
$$

Then we have

$$
\psi\left(x_{1}, x_{2}, t\right)=\psi_{1}\left(x_{1}, t\right) \psi_{2}\left(x_{2},-t\right),
$$

where $\psi_{1}\left(x_{1}, t\right)$ and $\psi_{2}\left(x_{2}, t\right)$ separately satisfy the Schrödinger equation with Hamiltonians $\hat{H}_{1}$ and $\hat{H}_{2}$ respectively. According to (6) (one should be careful with the gravitational acceleration term which is $\frac{g}{\sqrt{2}}$ in $\hat{H}_{1}$, but $-\frac{g}{\sqrt{2}}$ in $\left.\hat{H}_{2}\right)$,

$$
\begin{aligned}
& \psi_{1}\left(x_{1}, t\right)=\Phi_{1}\left(x_{1}+\frac{g t^{2}}{2 \sqrt{2}}, t\right) \exp \left[\frac{-i m g t}{\sqrt{2} \hbar}\left(x_{1}+\frac{g t^{2}}{6 \sqrt{2}}\right)\right], \\
& \psi_{2}\left(x_{2}, t\right)=\Phi_{2}\left(x_{2}-\frac{g t^{2}}{2 \sqrt{2}}, t\right) \exp \left[\frac{i m g t}{\sqrt{2} \hbar}\left(x_{2}-\frac{g t^{2}}{6 \sqrt{2}}\right)\right] .
\end{aligned}
$$

Thus (27) takes the form

$$
\psi(x, X, t)=e^{\frac{-i m g t}{\hbar} \frac{x_{1}+x_{2}}{\sqrt{2}}} \Phi_{1}\left(x_{1}+\frac{g t^{2}}{2 \sqrt{2}}, t\right) \Phi_{2}\left(x_{2}-\frac{g t^{2}}{2 \sqrt{2}},-t\right) .
$$

But $\frac{x_{1}+x_{2}}{\sqrt{2}}=X$ and

$$
\begin{gathered}
\Phi_{1}\left(x_{1}+\frac{g t^{2}}{2 \sqrt{2}}, t\right) \Phi_{2}\left(x_{2}-\frac{g t^{2}}{2 \sqrt{2}},-t\right)= \\
e^{\frac{i}{\hbar} \frac{g t^{2}}{2} \frac{\hat{p}_{1}-\hat{p}_{2}}{\sqrt{2}}} \Phi_{1}\left(x_{1}, t\right) \Phi_{2}\left(x_{2},-t\right)=e^{\frac{i}{\hbar} \frac{g t^{2}}{2} \hat{P}} \Phi(x, X, t)=\Phi\left(x+\frac{g t^{2}}{2}, X, t\right),
\end{gathered}
$$


and we reproduce again (16). "I have said it thrice: What I tell you three times is true" [29].

\section{Propagators in quantum and $\mathrm{KvN}$ mechanics}

Green functions or propagators are well-know useful tools in physics. The propagator $G\left(x_{f}, t_{f} ; x_{i}, t_{i}\right)$ is the probability amplitude for finding the particle at the position $x_{f}$ at time $t_{f}$, if at time $t_{i}$ it was at the position $x_{i}$. It can be calculated in a variety of ways, especially for Lagrangians which are, at most, quadratic in $x$ [30, 31, 32, 33, 34]. For this restricted class of problems, we can use the Fujiwara's formula [30]

$$
G\left(x_{f}, t_{f} ; x_{i}, t_{i}\right)=\sqrt{\frac{i}{2 \pi \hbar} \frac{\partial^{2} S\left(x_{f}, t_{f} ; x_{i}, t_{i}\right)}{\partial x_{i} \partial x_{f}}} \exp \left[\frac{i}{\hbar} S\left(x_{f}, t_{f} ; x_{i}, t_{i}\right)\right],
$$

where $S\left(x_{f}, t_{f} ; x_{i}, t_{i}\right)$ is the classical action.

For a free particle,

$$
S\left(x_{f}, t_{f} ; x_{i}, t_{i}\right)=\frac{m v^{2}}{2}\left(t_{f}-t_{i}\right)=\frac{m}{2} \frac{\left(x_{f}-x_{i}\right)^{2}}{t_{f}-t_{i}}
$$

and (31) gives the corresponding propagator

$$
G_{0}\left(x_{f}, t_{f} ; x_{i}, t_{i}\right)=\sqrt{\frac{m}{2 \pi i \hbar\left(t_{f}-t_{i}\right)}} \exp \left[\frac{i m\left(x_{f}-x_{i}\right)^{2}}{2 \hbar\left(t_{f}-t_{i}\right)}\right] .
$$

In the case of a freely falling particle, the solution of the equation of motion $\ddot{x}=-g$ with the boundary conditions $x\left(t_{i}\right)=x_{i}$ and $x\left(t_{f}\right)=x_{f}$ can be written in the following form [35]

$$
x-x_{0}=-\frac{1}{2} g\left(t-t_{0}\right)^{2},
$$

where

$$
\begin{aligned}
& t_{0}=\frac{t_{i}+t_{f}}{2}+\frac{1}{g} \frac{x_{f}-x_{i}}{t_{f}-t_{i}} \\
& x_{0}=\frac{x_{i}+x_{f}}{2}+\frac{g}{2}\left[\left(\frac{t_{f}-t_{i}}{2}\right)^{2}+\frac{1}{g^{2}}\left(\frac{x_{f}-x_{i}}{t_{f}-t_{i}}\right)^{2}\right] .
\end{aligned}
$$


Eq.(34) reveals a rarely noted property of uniqueness of free fall: each solution of $\ddot{x}=-g$ can be obtained by a spacetime translation of the primitive solution $x=-\frac{1}{2} g t^{2}$. Neither a free particle (relations (35) are singular at $g=0$ ), nor a harmonic oscillator share this interesting property [35].

From (34), $\dot{x}=-g\left(t-t_{0}\right)$. Thus $\frac{m}{2} \dot{x}^{2}=-m g\left(x-x_{0}\right)=\frac{1}{2} m g^{2}\left(t-t_{0}\right)^{2}$, and the action integral equals to

$$
\begin{aligned}
& S=\int_{t_{i}}^{t_{f}}\left[\frac{m}{2} \dot{x}^{2}-m g x\right] d t=\int_{t_{i}}^{t_{f}}\left[\frac{m}{2} \dot{x}^{2}-m g\left(x-x_{0}\right)-m g x_{0}\right] d t= \\
& \frac{1}{3} m g^{2}\left[\left(t_{f}-t_{0}\right)^{3}-\left(t_{i}-t_{0}\right)^{3}\right]-m g x_{0}\left(t_{f}-t_{i}\right) .
\end{aligned}
$$

Substituting $t_{0}$ and $x_{0}$ from (35), we finally obtain

$$
S\left(x_{f}, t_{f} ; x_{i}, t_{i}\right)=\frac{m\left(x_{f}-x_{i}\right)^{2}}{2\left(t_{f}-t_{i}\right)}-\frac{1}{2} m g\left(x_{i}+x_{f}\right)\left(t_{f}-t_{i}\right)-\frac{1}{24} m g^{2}\left(t_{f}-t_{i}\right)^{3},
$$

and then the Fujiwara's formula (31) gives the corresponding propagator:

$$
\begin{gathered}
G\left(x_{f}, t_{f} ; x_{i}, t_{i}\right)=\sqrt{\frac{m}{2 \pi i \hbar\left(t_{f}-t_{i}\right)}} \times \\
\exp \left\{\frac{i}{\hbar}\left[\frac{m\left(x_{f}-x_{i}\right)^{2}}{2\left(t_{f}-t_{i}\right)}-\frac{1}{2} m g\left(x_{i}+x_{f}\right)\left(t_{f}-t_{i}\right)-\frac{1}{24} m g^{2}\left(t_{f}-t_{i}\right)^{3}\right]\right\} .
\end{gathered}
$$

One can expect from the equivalence principle that $G$ and $G_{0}$ propagators are connected to each other in a simple way. This is indeed the case. From (6),$\psi(x, 0)=\phi(x, 0)$. Therefore,

$$
\psi(x, t)=\int_{-\infty}^{\infty} G(x, t ; y, 0) \psi(y, 0) d y=\int_{-\infty}^{\infty} G(x, t ; y, 0) \phi(y, 0) d y .
$$

On the other hand, again using ([6), we can write

$$
\begin{gathered}
\psi(x, t)=e^{-\frac{i m g t}{\hbar}\left(x+\frac{g t^{2}}{6}\right)} \phi\left(x+\frac{g t^{2}}{2}, t\right)= \\
e^{-\frac{i m g t}{\hbar}\left(x+\frac{g t^{2}}{6}\right)} \int_{-\infty}^{\infty} G_{0}\left(x+\frac{1}{2} g t^{2}, t ; y, 0\right) \phi(y, 0) d y .
\end{gathered}
$$


Comparing (40) and (39), we see that 34]

$$
G\left(x_{f}, t_{f} ; x_{i}, 0\right)=e^{-\frac{i}{\hbar} m g t_{f}\left(x_{f}+\frac{g t_{f}^{2}}{6}\right)} G_{0}\left(x_{f}+\frac{1}{2} g t_{f}^{2}, t_{f} ; x_{i}, 0\right) .
$$

Because of translational invariance in time, (41) can be written in a slightly more general form

$$
G\left(x_{f}, t_{f} ; x_{i}, t_{i}\right)=e^{-\frac{i}{\hbar} m g\left(t_{f}-t_{i}\right)\left(x_{f}+\frac{g\left(t_{f}-t_{i}\right)^{2}}{6}\right)} G_{0}\left(x_{f}+\frac{1}{2} g\left(t_{f}-t_{i}\right)^{2}, t_{f} ; x_{i}, t_{i}\right) \text {. }
$$

The validity of this relationship can be easily verified using (33) and (38).

To find propagators in KvN mechanics, the simplest way is to use the canonical transformation (25) (with unit Jacobian) and the relation (27). Then, on the one hand, we will have

$$
\begin{aligned}
& \psi\left(x_{f}, X_{f}, t_{f}\right)=\iint G^{(K v N)}\left(x_{f}, X_{f}, t_{f} ; x_{i}, X_{i}, t_{i}\right) \psi\left(x_{i}, X_{i}, t_{i}\right) d x_{i} d X_{i}= \\
& \iint G^{(K v N)}\left(x_{f}, X_{f}, t_{f} ; x_{i}, X_{i}, t_{i}\right) \psi_{1}\left(x_{1 i}, t_{i}\right) \psi_{2}\left(x_{2 i},-t_{i}\right) d x_{1 i} d x_{2 i} .
\end{aligned}
$$

On the other hand,

$$
\begin{gathered}
\psi\left(x_{f}, X_{f}, t_{f}\right)=\psi_{1}\left(x_{1 f}, t_{f}\right) \psi_{2}\left(x_{2 f},-t_{f}\right)= \\
\iint G\left(x_{1 f}, t_{f} ; x_{1 i}, t_{i}\right) G\left(x_{2 f},-t_{f} ; x_{2 i},-t_{i}\right) \psi_{1}\left(x_{1 i}, t_{i}\right) \psi_{2}\left(x_{2 i},-t_{i}\right) d x_{1 i} d x_{2 i} .
\end{gathered}
$$

Comparing, we see that

$$
G^{(K v N)}\left(x_{f}, X_{f}, t_{f} ; x_{i}, X_{i}, t_{i}\right)=G\left(x_{1 f}, t_{f} ; x_{1 i}, t_{i}\right) G\left(x_{2 f},-t_{f} ; x_{2 i},-t_{i}\right) .
$$

Since $\left(x_{1 f}-x_{1 i}\right)^{2}+\left(x_{2 f}-x_{2 i}\right)^{2}=\left(x_{1 f}-x_{2 f}-x_{1 i}+x_{2 i}\right)\left(x_{1 f}+x_{2 f}-x_{1 i}-x_{2 i}\right)=$ $2\left(x_{f}-x_{i}\right)\left(X_{f}-X_{i}\right)$, from (45) we obtain a free KvN-propagator

$$
G_{0}^{(K v N)}\left(x_{f}, X_{f}, t_{f} ; x_{i}, X_{i}, t_{i}\right)=\frac{m}{2 \pi \hbar\left(t_{f}-t_{i}\right)} \exp \left[\frac{i m\left(x_{f}-x_{i}\right)\left(X_{f}-X_{i}\right)}{\hbar\left(t_{f}-t_{i}\right)}\right] .
$$

Analogously, from (38) and (45), and remembering that the gravitational acceleration terms are $g / \sqrt{2}$ and $-g / \sqrt{2}$ for $x_{1}$ and $x_{2}$ respectively, we get a KvN-propagator in the homogeneous gravitational field:

$$
\begin{gathered}
G^{(K v N)}\left(x_{f}, X_{f}, t_{f} ; x_{i}, X_{i}, t_{i}\right)= \\
\frac{m}{2 \pi \hbar\left(t_{f}-t_{i}\right)} \exp \left[\frac{i}{\hbar}\left\{\frac{m}{t_{f}-t_{i}}\left(x_{f}-x_{i}\right)\left(X_{f}-X_{i}\right)-\frac{m g}{2}\left(X_{f}+X_{i}\right)\left(t_{f}-t_{i}\right)\right\}\right] .
\end{gathered}
$$


As in the quantum case, there is a relation between the free $\mathrm{KvN}$ propagator and the $\mathrm{KvN}$ propagator in a uniform gravitational field, as follows from the principle of equivalence. To obtain this relation, we will disentangle noncommutative operators in the expression for the $\mathrm{KvN}$ propagator in a uniform gravitational field 31]

$$
G^{(K v N)}\left(x_{f}, X_{f}, t_{f} ; x_{i}, X_{i}, t_{i}\right)=\left\langle x_{f}, X_{f}\left|e^{-\frac{i}{\hbar}\left(\frac{\hat{p} \hat{P}}{m}+m g \hat{X}\right)\left(t_{f}-t_{i}\right)}\right| x_{i}, X_{i}\right\rangle .
$$

Namely, we have (compare with (15))

$$
e^{-\frac{i}{\hbar}\left(\frac{\hat{p} \hat{P}}{m}+m g \hat{X}\right)\left(t_{f}-t_{i}\right)}=e^{\frac{i}{\hbar} \frac{g\left(t_{f}-t_{i}\right)^{2}}{2}} \hat{P} e^{-\frac{i}{\hbar} m g \hat{X}\left(t_{f}-t_{i}\right)} e^{-\frac{i}{\hbar} \frac{\hat{p} \hat{P}}{m}\left(t_{f}-t_{i}\right)} .
$$

But

$$
\begin{aligned}
& \left\langle x_{f}, X_{f}\right| e^{\frac{i}{\hbar} \frac{g\left(t_{f}-t_{i}\right)^{2}}{2} \hat{P}}=\left\langle x_{f}+\frac{1}{2} g\left(t_{f}-t_{i}\right)^{2}, X_{f}\right|, \\
& \left\langle x_{f}, X_{f}\right| e^{-\frac{i}{\hbar} m g \hat{X}\left(t_{f}-t_{i}\right)}=e^{-\frac{i}{\hbar} m g X_{f}\left(t_{f}-t_{i}\right)}\left\langle x_{f}, X_{f}\right|,
\end{aligned}
$$

and

$$
G_{0}^{(K v N)}\left(x_{f}, X_{f}, t_{f} ; x_{i}, X_{i}, t_{i}\right)=\left\langle x_{f}, X_{f}\left|e^{-\frac{i}{\hbar} \frac{\hat{p} \hat{P}}{m}\left(t_{f}-t_{i}\right)}\right| x_{i}, X_{i}\right\rangle
$$

is the free $\mathrm{KvN}$-propagator. Therefore, (49) implies the following relationship:

$$
\begin{gathered}
G^{(K v N)}\left(x_{f}, X_{f}, t_{f} ; x_{i}, X_{i}, t_{i}\right)= \\
e^{-\frac{i}{\hbar} m g X_{f}\left(t_{f}-t_{i}\right)} G_{0}^{(K v N)}\left(x_{f}+\frac{1}{2} g\left(t_{f}-t_{i}\right)^{2}, X_{f}, t_{f} ; x_{i}, X_{i}, t_{i}\right),
\end{gathered}
$$

similar to (42). The validity of this relation can be easily verified using (46) and (47).

It is rather instructive to obtain KvN propagator (47) by Schwinger's method [32]. First of all, since the propagator depends only on the difference $\tau=t_{f}-t_{i}$, and not separately on $t_{f}$ and $t_{i}$, let's introduce slightly more compact notation:

$$
G^{(K v N)}\left(x_{f}, X_{f} ; x_{i}, X_{i} ; \tau\right)=\left\langle x_{f}, X_{f}\left|e^{-\frac{i}{\hbar} \hat{H} \tau}\right| x_{i}, X_{i}\right\rangle=\left\langle x_{f}, X_{f}, \tau \mid x_{i}, X_{i}, 0\right\rangle .
$$

From (53), we get a differential equation

$$
\begin{aligned}
& i \hbar \frac{\partial}{\partial \tau} G^{(K v N)}\left(x_{f}, X_{f} ; x_{i}, X_{i} ; \tau\right)=\left\langle x_{f}, X_{f}\left|\hat{H} e^{-\frac{i}{\hbar} \hat{H} \tau}\right| x_{i}, X_{i}\right\rangle= \\
& \left\langle x_{f}, X_{f}, \tau|\hat{H}| x_{i}, X_{i}, 0\right\rangle=\left\langle x_{f}, X_{f}, \tau\left|m^{-1} \hat{p}(0) \hat{P}(0)+m g \hat{X}(0)\right| x_{i}, X_{i}, 0\right\rangle .
\end{aligned}
$$


In (53) and (54) we have introduced notations

$$
|x, X, t\rangle=e^{\frac{i}{\hbar} \hat{H} t}|x, X\rangle, \quad \hat{x}(t)=e^{\frac{i}{\hbar} \hat{H} t} \hat{x} e^{-\frac{i}{\hbar} \hat{H} t}, \hat{X}(t)=e^{\frac{i}{\hbar} \hat{H} t} \hat{X} e^{-\frac{i}{\hbar} \hat{H} t},
$$

and analogously for $\hat{p}(t)$ and $\hat{P}(t)$. Note that $|x, X, t\rangle$ is a common eigenstate of $\hat{x}(t)$ and $\hat{X}(t)$. Therefore, one can easily calculate the right-hand side of (54), if $\hat{p}(0) \hat{P}(0)$ can be written in terms of $\hat{x}(\tau), \hat{X}(\tau), \hat{x}(0)$ and $\hat{X}(0)$ in such a way that $\hat{x}(\tau)$ and $\hat{X}(\tau)$ appear on the left-hand side, while $\hat{x}(0)$ and $\hat{X}(0)$ appear on the right-hand side. This can be achieved with the help of Heisenberg equation of motion $i \hbar \frac{d \hat{A}}{d t}=[\hat{A}, \hat{H}]$, which gives

$$
\frac{d \hat{x}}{d t}=\frac{\hat{p}}{m}, \quad \frac{d \hat{p}}{d t}=-m g, \quad \frac{d \hat{X}}{d t}=\frac{\hat{P}}{m}, \quad \frac{d \hat{P}}{d t}=0 .
$$

The solutions of these equations that we need are as follows:

$$
\hat{x}(t)=\hat{x}(0)+\frac{\hat{p}(0)}{m} t-\frac{1}{2} g t^{2}, \quad \hat{X}(t)=\hat{X}(0)+\frac{\hat{P}(0)}{m} t .
$$

Therefore,

$$
\hat{p}(0)=\frac{m}{\tau}\left[\hat{x}(\tau)-\hat{x}(0)+\frac{1}{2} g \tau^{2}\right], \quad \hat{P}(0)=\frac{m}{\tau}[\hat{X}(\tau)-\hat{X}(0)],
$$

and we get

$$
\begin{gathered}
\hat{H}=\frac{1}{m} \hat{p}(0) \hat{P}(0)+m g \hat{X}(0)=\frac{1}{2} m g(\hat{X}(\tau)+\hat{X}(0))- \\
i \hbar \frac{1}{\tau}+\frac{m}{\tau^{2}}[\hat{X}(\tau)(\hat{x}(\tau)-\hat{x}(0))-(\hat{x}(\tau)-\hat{x}(0)) \hat{X}(0)] .
\end{gathered}
$$

To get the correct time-ordering of operators in (59), we have used the relation $\hat{x}(0) \hat{X}(\tau)=[\hat{x}(0), \hat{X}(\tau)]+\hat{X}(\tau) \hat{x}(0)$, and

$$
[\hat{x}(0), \hat{X}(\tau)]=\left[\hat{x}(0), \hat{X}(0)+\frac{\hat{P}(0)}{m} \tau\right]=i \hbar \frac{\tau}{m} .
$$

Substituting (59) into (54), we get

$$
i \hbar \frac{\partial G^{(K v N)}}{\partial \tau}=\left[\frac{m}{\tau^{2}}\left(x_{f}-x_{i}\right)\left(X_{f}-X_{i}\right)-i \hbar \frac{1}{\tau}+\frac{1}{2} m g\left(X_{f}+X_{i}\right)\right] G^{(K v N)} .
$$


This differential equation can be easily integrated with the result

$$
G^{(K v N)}\left(x_{f}, X_{f} ; x_{i}, X_{i} ; \tau\right)=\frac{C}{\tau} e^{\frac{i}{\hbar}\left[\frac{m}{\tau}\left(x_{f}-x_{i}\right)\left(X_{f}-X_{i}\right)-\frac{m g}{2}\left(X_{f}+X_{i}\right) \tau\right]},
$$

where $C$ is an integration constant. To determine $C$, we use the initial condition

$$
G^{(K v N)}\left(x_{f}, X_{f} ; x_{i}, X_{i} ; 0\right)=\left\langle x_{f}, X_{f}, 0 \mid x_{i}, X_{i}, 0\right\rangle=\delta\left(x_{f}-x_{i}\right) \delta\left(X_{f}-X_{i}\right) .
$$

Using [36]

$$
\lim _{\tau \rightarrow 0} \sqrt{\frac{1}{\pi \tau}} e^{-\frac{1}{\tau} x^{2}}=\delta(x),
$$

and $\left(x_{f}-x_{i}\right)\left(X_{f}-X_{i}\right)=\frac{1}{2}\left[\left(x_{1 f}-x_{1 i}\right)^{2}-\left(x_{2 f}-x_{2 i}\right)^{2}\right]$, which follows from (25), we get

$$
\lim _{\tau \rightarrow 0} \frac{1}{\tau} e^{\frac{i}{\hbar}\left[\frac{m}{\tau}\left(x_{f}-x_{i}\right)\left(X_{f}-X_{i}\right)\right]}=\frac{2 \pi \hbar}{m} \delta\left(x_{2 f}-x_{2 i}\right) \delta\left(x_{1 f}-x_{1 i}\right) .
$$

But, since the Jacobian of the transformation $(x, X) \rightarrow\left(x_{1}, x_{2}\right)$, implied by (25), is unity, $\delta\left(x_{2 f}-x_{2 i}\right) \delta\left(x_{1 f}-x_{1 i}\right)=\delta\left(x_{2}-x_{i}\right) \delta\left(X_{f}-X_{i}\right)$ [36], and finally we obtain $C=\frac{m}{2 \pi \hbar}$. Therefore, (62) is the same as (47).

\section{Free fall in quantum and $\mathrm{KvN}$ mechanics}

One can expect a thorough consideration of the quantum free fall in standard textbooks on quantum mechanics, but, surprisingly, this is not so. Some aspects of this problem was considered in [37, 38], and one can find a rather exhaustive account in unpublished notes [35].

Here we are interested in the following question: is it possible to obtain information about the mass of a freely falling quantum or $\mathrm{KvN}$ probe by performing coordinate measurements on the probe during the free fall?

It can be argued that a quantitative measure of the amount of information about the mass of the probe we can obtain by performing measurements of its coordinate $x$ is provided by Fisher's information [39]

$$
I_{m}=\int d x|\psi(x, t)|^{2}\left[\frac{\partial}{\partial m} \ln |\psi(x, t)|^{2}\right]^{2} \text {. }
$$


Fisher information in general is a measure of the ability to estimate an unknown parameter and is an important concept of mathematical statistics with many physical applications [40].

Suppose we are dropping the following initial Gaussian wave packet

$$
\psi(x, 0)=\left(\frac{2}{\pi a^{2}}\right)^{\frac{1}{4}} \exp \left[-\frac{x^{2}}{a^{2}}+\frac{i}{\hbar} p x\right] .
$$

In the momentum space

$$
\psi(k)=\frac{1}{\sqrt{2 \pi \hbar}} \int_{-\infty}^{\infty} e^{-\frac{i}{\hbar} k x} \psi(x, 0) d x=\left(\frac{a^{2}}{2 \pi \hbar^{2}}\right)^{\frac{1}{4}} \exp \left[-\frac{a^{2}}{4 \hbar^{2}}(k-p)^{2}\right] .
$$

Therefore, (67) describes a Gaussian wave packet centered around zero in the configuration space and centered around $p$ in the momentum space. To find $\psi(x, t)$, we can use (6) and time-evolved free wave-packet [41]

$$
\phi(x, t)=\frac{1}{\sqrt{\alpha}}\left(\frac{a^{2}}{8 \pi}\right)^{\frac{1}{4}} \exp \left[\frac{i}{\hbar} p\left(x-\frac{p t}{2 m}\right)\right] \exp \left[-\frac{1}{4 \alpha}\left(x-\frac{p t}{m}\right)^{2}\right]
$$

where $\alpha=\frac{a^{2}}{4}+\frac{i \hbar t}{2 m}$. Then

$$
|\psi(x, t)|^{2}=\left|\phi\left(x+\frac{1}{2} g t^{2}, t\right)\right|^{2}
$$

with [41]

$$
|\phi(x, t)|^{2}=\frac{1}{\sqrt{2 \pi} \Delta(t)} \exp \left[-\frac{\left(x-\frac{p t}{m}\right)^{2}}{2[\Delta(t)]^{2}}\right], \quad \Delta(t)=\frac{a}{2} \sqrt{1+\frac{4 \hbar^{2} t^{2}}{m^{2} a^{4}}} .
$$

First of all note that, because of (70), the expectation value of $x$ turns out to be

$$
\langle x\rangle=\int_{-\infty}^{\infty} x|\psi(x, t)|^{2} d x=\int_{-\infty}^{\infty}\left(y-\frac{1}{2} g t^{2}\right)|\phi(y, t)|^{2} d y=\frac{p t}{m}-\frac{1}{2} g t^{2} .
$$

Therefore the wave packet (67) indeed describes a freely falling quantum probe (with initial momentum $p$ ). 
The second consequence of (70) is that the Fisher information related to free fall is the same as for a free particle (we can make a change of integration variable from $x$ to $y=x+\frac{1}{2} g t^{2}$ in (66) $)$. Using

$$
\frac{\partial \Delta(t)}{\partial m}=-\frac{\hbar^{2} t^{2}}{a^{2} m^{3}} \frac{1}{\Delta(t)}
$$

and

$$
\frac{\partial \ln |\phi(x, t)|^{2}}{\partial m}=\frac{\hbar^{2} t^{2}}{a^{2} m^{3}}\left(\frac{1}{\Delta(t)^{2}}-\frac{\left(x-\frac{p t}{m}\right)^{2}}{\Delta(t)^{4}}\right)-\frac{x-\frac{p t}{m}}{\Delta(t)^{2}} \frac{p t}{m^{2}},
$$

it is straightforward to get

$$
I_{m}=\frac{1}{m^{2}}\left[\frac{2}{\left[1+\left(\frac{m a^{2}}{2 \hbar t}\right)^{2}\right]^{2}}+\left(\frac{p a}{\hbar}\right)^{2} \frac{1}{1+\left(\frac{m a^{2}}{2 \hbar t}\right)^{2}}\right] .
$$

As we see, when $t \rightarrow \infty$, the Fisher information is saturated to a constant value, which means that by monitoring a freely-falling quantum probe, one cannot arbitrarily improve the accuracy of determining its mass [39]. Anyway, this Fisher information doesn't depend on $g$. A homogeneous gravitational field does not allow obtaining more information about the mass of a freely falling quantum probe than is possible using a free quantum probe. This constitutes a possible formulation of the weak principle of equivalence in the quantum realm [39].

Now consider a KvN particle. In the role of the initial wave packet, we will take a double Gaussian

$$
\psi(x, p, 0)=\sqrt{\frac{2}{\pi a b}} \exp \left[-\frac{x^{2}}{a^{2}}-\frac{\left(p-p_{0}\right)^{2}}{b^{2}}\right] .
$$

This wave function is in the $(x, p)$-representation where its physical meaning is especially clear. As we already know, the Koopman Koopman-von Neumann mechanics is the most quantum-like in the $(x, X)$-representation. In this representation (176) takes the form

$$
\begin{gathered}
\psi(x, X, 0)=\frac{1}{\sqrt{2 \pi \hbar}} \int_{-\infty}^{\infty} e^{\frac{i}{\hbar} p X} \psi(x, p, 0) d p= \\
\sqrt{\frac{b}{a \pi \hbar}} \exp \left[-\frac{x^{2}}{a^{2}}-\frac{X^{2} b^{2}}{4 \hbar^{2}}+\frac{i}{\hbar} p_{0} X\right] .
\end{gathered}
$$


In the homogeneous gravitational field, time-evolved wave function $\psi(x, X, t)$ can be found by using (16) and the time-evolved free wave packet. To find the later, it is convenient first to find a time-evolved free wave packet in the $(x, p)$-representation. Free propagator in this representation has the following form [42, 43]

$$
\begin{aligned}
& G_{0}^{(K v N)}\left(x_{f}, p_{f} ; x_{i}, p_{i} ; \tau\right)=\left\langle x_{f}, p_{f}\left|e^{-\frac{i}{\hbar} \frac{\hat{p} \hat{p}}{m} \tau}\right| x_{i}, p_{i}\right\rangle= \\
& \left\langle x_{f}, p_{f} \mid x_{i}+\frac{p_{i}}{m} \tau, p_{i}\right\rangle=\delta\left(x_{f}-x_{i}-\frac{p_{i}}{m} \tau\right) \delta\left(p_{f}-p_{i}\right) .
\end{aligned}
$$

Therefore, the time-evolved form of (76) under free evolution is [42]

$$
\phi(x, p, t)=\sqrt{\frac{2}{\pi a b}} \exp \left[-\frac{1}{a^{2}}\left(x-\frac{p}{m} t\right)^{2}-\frac{\left(p-p_{0}\right)^{2}}{b^{2}}\right] .
$$

Then

$$
\begin{gathered}
\phi(x, X, t)=\frac{1}{\sqrt{2 \pi \hbar}} \int_{-\infty}^{\infty} e^{\frac{i}{\hbar} p X} \psi(x, p, t) d p= \\
\sqrt{\frac{m^{2} a b}{\pi \hbar\left(m^{2} a^{2}+b^{2} t^{2}\right)}} \exp \left[-\frac{x^{2}}{a^{2}}-\frac{p_{0}^{2}}{b^{2}}-\frac{\left(i b^{2} t x+i p_{0} m a^{2}-\frac{m a^{2} b^{2}}{2 \hbar} X\right)^{2}}{a^{2} b^{2}\left(m^{2} a^{2}+t^{2} b^{2}\right)}\right]
\end{gathered}
$$

and a simple calculation shows that

$$
|\phi(x, X, t)|^{2}=\frac{a b}{4 \pi \hbar \Delta^{2}(t)} \exp \left[-\frac{1}{2 \Delta^{2}(t)}\left[\left(x-\frac{p_{0}}{m} t\right)^{2}+\frac{a^{2} b^{2}}{4 \hbar^{2}} X^{2}\right]\right],
$$

where

$$
\Delta(t)=\frac{a}{2} \sqrt{1+\frac{b^{2} t^{2}}{m^{2} a^{2}}} .
$$

Using (16) and (81), we can easily find that the expectation value of $x$ in the state $\psi(x, X, t)$ is

$$
\langle x\rangle=\int_{-\infty}^{\infty} \int_{-\infty}^{\infty} x|\psi(x, X, t)|^{2} d x d X=\frac{p_{0} t}{m}-\frac{1}{2} g t^{2} .
$$

Since $X$ is a hidden variable, when calculating Fisher information, instead of $|\psi(x, X, t)|^{2}$ we should use the following probability density

$$
\rho(x, t)=\int_{-\infty}^{\infty}|\psi(x, X, t)|^{2} d X=\int_{-\infty}^{\infty}\left|\phi\left(x+\frac{g t^{2}}{2}, X, t\right)\right|^{2} d X .
$$


But

$$
I_{m}=\int_{-\infty}^{\infty} \rho(x, t)\left[\frac{\partial}{\partial m} \ln \rho(x, t)\right]^{2} d x=\int_{-\infty}^{\infty} \rho(y, t)\left[\frac{\partial}{\partial m} \ln \rho(y, t)\right]^{2} d y
$$

where $y=x+\frac{g t^{2}}{2}$, and therefore

$$
\rho(y, t)=\int_{-\infty}^{\infty}|\phi(y, X, t)|^{2} d X=\frac{1}{\sqrt{2 \pi} \Delta(t)} \exp \left[-\frac{1}{2 \Delta^{2}(t)}\left(y-\frac{p_{0}}{m} t\right)^{2}\right] .
$$

Surprisingly enough, (71) turns out to be a special case of (86) with $b=\frac{2 \hbar}{a}$, as (68) indicates. Therefore, the corresponding Fisher information can be obtained from (75) by substitution $2 \hbar=a b$ :

$$
I_{m}=\frac{1}{m^{2}}\left[\frac{2}{\left[1+\left(\frac{m a}{b t}\right)^{2}\right]^{2}}+\left(\frac{2 p_{0}}{b}\right)^{2} \frac{1}{1+\left(\frac{m a}{b t}\right)^{2}}\right] .
$$

The behaviour of $I_{m}$, if $b \neq 0$, is similar to the quantum case. Note that $I_{m}=0$ if $p_{0}=b=0$, but $a \neq 0$. Therefore, if an assembly of identical classical particles with strictly zero initial momentum, but some dispersion in initial positions, is dropped in the uniform gravitational field, we cannot determine the mass of the particles by observing evolution of their positions in time. However, if $p_{0} \neq 0, a \neq 0$, but $b=0$, we will have

$$
I_{m}=\frac{4 p_{0}^{2} t^{2}}{m^{4} a^{2}}
$$

Therefore, if we know precisely the initial momentum of particles, observing their positions will allow us to find their mass, and our ability to do this more precisely will increase quadratically in time.

If in addition $a \rightarrow 0$, then $\Delta(t) \rightarrow 0$ and it follows from (64) and (86) that $\rho(x, t)=\delta\left(x+\frac{g t^{2}}{2}-\frac{p_{0}}{m} t\right)$ that describes a classical particle (not an assembly of identical particles) dropped in a uniform gravitational field with some initial momentum $p_{0}$. In this case, the Fisher information (88) diverges: if there is no uncertainty in the initial position of the particle, a single measurement of its position at some later time will be sufficient to find its initial velocity and hence the mass, if the initial momentum is known. 
We can use (79) to calculate Fisher information in the case, when we perform momentum measurements in addition to the coordinate measurements. The result is similar to (88) (and coincides to it when $b=0$ ):

$$
I_{m}=\frac{t^{2}}{m^{4} a^{2}}\left[b^{2}+4 p_{0}^{2}\right] .
$$

\section{Concluding remarks}

Implementation of Einstein's principle of equivalence in $\mathrm{KvN}$ mechanics, as shown in this article, is strikingly similar to its implementation in quantum mechanics. Therefore, the belief that is sometimes found in the literature that the mass dependence of a quantum-mechanical wave function implies that the weak principle of equivalence is violated in quantum mechanics is erroneous: locally, in the limit of a uniform field, the weak principle of equivalence does hold in quantum mechanics in the same way as in classical mechanics, if the latter is formulated in the language of Hilbert space states and operators.

The independence of the Fisher information $I_{m}$ from the magnitude of the homogeneous gravitational field is a formulation of Einstein's principle of equivalence, which is universally applicable in both $\mathrm{KvN}$ and in quantum mechanics.

If the gravitational field is not homogeneous, Fisher information in quantum mechanics depends on curvature (tidal effects) and quantum wave function can thus imprint nontrivial information about the mass [39]. The same applies to $\mathrm{KvN}$ mechanics, although we did not address this issue in this article. This is hardly surprising, since it is well known in general relativity that a classical extended body can sense tidal forces and thus does not follow a geodesic in general [44].

Both in $\mathrm{KvN}$ mechanics and in quantum mechanics, the wave function in the freely falling reference frame differs from the free wave function only in phase. In contrast to quantum mechanics, however, in $\mathrm{KvN}$ mechanics this phase depends on the unobservable hidden variable $X$ and thus can not lead to the Colella-Overhauser-Werner type interference effects observed in quantum mechanics, since we have no control on the variable $X$ and cannot organize the splitting and recombination of the particle beam in the $X$ direction 1 .

\footnotetext{
${ }^{1}$ Planck-scale quantum gravity effects can destroy unobservability of hidden variables
} 


\section{Appendix A. Free fall: a historical perspective}

When it comes to remembering Galileo, the first glimpse that most people will have is the portrait where he is shown to drop objects of various masses from the Leaning tower of Pisa [45]. There is a good reason to believe that this is merely a legend, and in fact, Galileo never did this experiment: due to air resistance, such an experiment, if performed, clearly shows that heavy objects fall faster than light objects [46, 47, 48], in full confirmation of the views of Aristotle (in fact, it can be proved [48] that in arbitrary resistive medium the fall time decreases with mass). For example, in a reconstruction of the Galileo's alleged Leaning Tower of Pisa experiment a 16-pound shot and a softball were dropped from the tower approximately from the 200 feet height, and the shot reached the ground 20-30 feet ahead of the softball [46].

However, it appears that Galileo did indeed perform some other experiments skillfully using inclined planes to dilute the gravitational acceleration, thereby reducing speeds, as well as the associated air resistance involved in the experiments. Although there are grave doubts among professional historians of science whether Galileo ever performed any significant experiments at all [49], in the case of experiments with an inclined plane, it was at least demonstrated that Galileo's alleged experimental results can be reproduced using devices that are very similar to those described by Galileo [50, 51].

One way or another, regardless of the role that real experiments played in Galileo's conclusions, these conclusions were quite interesting. Galileo asserted that the trajectory of a dropped mass, when it falls under the action of gravity, and its time of descent to the ground depend only on its initial position and velocity, and is independent of its weight. He argues several times in his writings that this property of falling bodies is a logical necessity that can be inferred from a certain thought experiment. However, there is a flaw in his argument that becomes especially obvious if we apply this argument to electric force, where it gives a clearly incorrect conclusion [52]. In fact, Galileo's universality of free fall is an empirical fact, and as such it can be refuted by experiment. For example, Lunar Laser Ranging data allows us to verify that Earth and Moon, despite their different masses and compositions, fall to the Sun with accelerations that are equal to each other with an accuracy of one part in 10 billion [53].

and thus $\mathrm{KvN}$ phase factors can potentially also lead to some physical effects, but the expected magnitude of such effects is vanishingly small [87]. 
Free fall plays a unique role in the history of science, as it has led to three major breakthroughs in our understanding of gravity. The first was Galileo's discovery of universality of free fall. The second breakthrough, the creation of Newton's theory of universal gravitation, is also associated with the legend. The legend says that a falling apple inspired Isaac Newton's discovery of the law of gravity. The origin of the story can be traced back to Newton himself who spoke about this a few months before his death to his younger friend William Stukeley [54, 55], and then told the same story to at least three other people on various occasions [55]. It is not clear why Newton attributed such significance to this story. Even if the falling apple really triggered Newton's musings on the nature of gravitation, the growth of theory from this seed was rather slow and complicated process influenced by Newton's interactions with other contemporary scientists [56, 57].

\section{Appendix B. Free fall and geometrization of gravity}

In modern terms, the main features of the magnificent edifice of Newtonian physics, whose foundations were laid by Newton, are the following [58, 59, 60, 61]. All material processes (events) are imagined to take place in spacetime - a 4-dimensional, real, smooth manifold $M$. Newtonian ab-

solute time $t$ is a smooth map with nonvanishing gradient (defined up to a linear transformations $t \rightarrow a t+b$ with $a>0$ ) from $M$ into the real line. Newtonian time defines the absolute (observer independent) simultaneity relation between events of $M$ and thus stratifies $M$ : the manifold is partitioned into space sections each consisting of a totality of simultaneous events. Each space section is assumed to have the structure of a Euclidean three dimensional space with a positive definite metric. In such Galilean manifold, one can distinguish between spacelike vectors (which lay completely in one space section) and future or past directed timelike vectors. A world-line of an observer is a timelike curve $C$ in $M$ and it can be used to introduce Galilean coordinates in $M$. Namely, for any event $P$, let $O$ be the simultaneous event on $C$. Then $t(P)=t(O)$ and spatial coordinates of $P$ are just Cartesian coordinates of $P$ in the space section containing $P$ and $O$, with $O$ as the origin of the Cartesian frame. Coordinates defined by different Galilean observers are related by the transformation

$$
x^{i}=R_{j}^{i}(t) x^{j}+\xi^{i}(t), \quad t^{\prime}=t+\tau,
$$


where $\xi^{i}$ is an arbitrary time-dependent vector, $\tau$ is a real number and $R_{j}^{i}$ is a real (time-dependent) orthogonal matrix. These transformations form the kinematical (Leibnizian in another terminology [62]) group $\mathcal{K}$. We assume that units for time and length are the same for all Galilean observers and therefore the above mentioned linear ambiguity in the definition of the Newtonian absolute time is reduced just to the freedom (reflected by the presence of $\tau$ in (B.1) $)$ of Galilean observers to choose different origins of their time axis.

Singling out a particular class of world-lines as standard motions, in such a way that there is one and only one standard world line through each event and time-like direction at that event, then determines the dynamics. Namely, standard motions are considered as force-free. For all other motions, forces are defined in terms of accelerations relative to the standard motions. The generalized Newton's second equation has the form

$$
m[\ddot{\vec{x}}-\vec{a}(\vec{x}, \dot{\vec{x}})]=\vec{F},
$$

where $\vec{F}$ is the force, $\vec{x}(t)$ describes the motion of a particle relative to some Galilean observer, and $\vec{a}(\vec{x}, \dot{\vec{x}})$ is the the acceleration due to a standard motion of the observer himself.

The Newtonian way of introducing a congruence of preferred world-lines (standard motions) is to postulate the validity of Newton's law of inertia (the first law): There exist the special class of Galilean observers (inertial frames of reference) with respect to which free particles have zero acceleration:

$$
\ddot{\vec{x}}=0 \text {. }
$$

The transformations (B.1) to preserve (B.3), one should have

$$
\dot{R}_{j}^{i}=0, \quad \ddot{\xi}^{i}=0 .
$$

Such kind of transformations constitute a subgroup of the kinematical group called the Galilei group $\mathcal{G} \subset \mathcal{K}$. Since, according to (B.1) and (B.4), Galilean transformations are linear, it is meaningful to call two Galilean 4-vectors parallel if they have the same components with respect to inertial coordinate systems. Mathematically Newton's first law defines on Galilean spacetime an integrable symmetric connection whose components all vanish in an inertial coordinate system. Timelike geodesics of this connection represent the free motions. 
So far so good, but how to empirically determine inertial reference systems? With some care, one can eliminate known forces acting on the test body, such as electric and magnetic forces, drag force, and etc. However, to eliminate gravity is not a simple task because even a neutral test body will accelerate towards massive objects. If one assumes that a test body, besides the inertial mass $m_{i}$, has a gravitational mass $m_{g}$, then Newton's second law in inertial reference frame takes the form

$$
m_{i} \ddot{\vec{x}}=m_{g} \vec{g}(\vec{x}, t)+\vec{F},
$$

where $\vec{g}$ is the gravitational field strength, and $\vec{F}$ is any additional nongravitational force acting on the test body. In arbitrary Galilean reference frame non-inertial (fictitious) forces will appear and (B.5) is replaced by

$$
\ddot{\vec{x}}=\frac{m_{g}}{m_{i}} \vec{g}+\frac{\vec{F}}{m_{i}}-\vec{a}-2 \vec{\omega} \times \dot{\vec{x}}-\dot{\vec{\omega}} \times \vec{x}-\vec{\omega} \times(\vec{\omega} \times \vec{x}),
$$

where $\vec{a}$ and $\vec{\omega}$ are, respectively, the acceleration and the angular velocity of the Galilean laboratory frame relative to some inertial frame.

Universality of free fall means that the ratio $m_{g} / m_{i}$ is a universal constant that can be taken equal to unity without loss of generality. Then (B.6) indicates that by observing the motions of particles relative to some laboratory frame, we can in principle measure $\vec{g}-\vec{a}$, but not $\vec{g}$ and $\vec{a}$ separately. Gravity and inertial acceleration are inseparably mingled. Because of this circumstance, the law of inertia should be replaced by the weak principle of equivalence: There exist free fall motions under which gravity is locally canceled by free fall acceleration.

Free fall motions define a special class of Galilean reference frames, the Newtonian frames, and if such motions are taken as the standard motions of dynamics, the Newton's second law in the Newtonian frames takes the form

$$
m(\ddot{\vec{x}}+\nabla \Phi)=\vec{F}
$$

where $\Phi(\vec{x}, t)$ is the gravitational potential related to the gravitational field strength by relation $\vec{g}=-\nabla \Phi$.

Comparing (B.7) and (B.6), we see that Newtonian frames are nonrotating Galilean frames (Coriolis and centrifugal accelerations are absent in the equation of motion of a test body). Therefore, the kinematical transformations (B.1) that relate different Newtonian frames are singled out by 
the condition that $R_{j}^{i}$ are time-independent: $\dot{R}_{j}^{i}=0$. Accordingly, the Newtonian group $\mathcal{N}$ of such transformations (it is called Maxwellian in [62]) is intermediate in generality between the Galilean group $\mathcal{G}$ and the kinematical group $\mathcal{K}: \mathcal{G} \subset \mathcal{N} \subset \mathcal{K}$.

Let's demand equation (B.7) to be form-invariant (covariant) with respect to Newtonian transformations. Due to vector character of (B.7), it is automatically covariant under rotations given by constant orthogonal matrix $R_{j}^{i}$ provided the gravitational potential is a scalar with respect to such transformations. The same is true for pure time translations $t^{\prime}=t+\tau$. Therefore, without loss of generality, we can consider special Newtonian transformations

$$
\vec{x}^{\prime}=\vec{x}+\vec{\xi}(t), \quad t^{\prime}=t .
$$

Then $\nabla^{\prime}=\nabla, \ddot{\vec{x}^{\prime}}=\ddot{\vec{x}}+\ddot{\vec{\xi}}$, and from (for zero external force) $\ddot{\vec{x}}^{\prime}-\nabla^{\prime} \Phi^{\prime}=$ $\ddot{\vec{x}}-\nabla \Phi=0$ we get

$$
\nabla\left(\Phi^{\prime}-\Phi\right)=-\ddot{\vec{\xi}}
$$

which shows that under (B.8) the gravitational potential is not a scalar but transforms according to (note that we should have $\Phi^{\prime}=\Phi$ when $\vec{\xi}=0$ )

$$
\Phi^{\prime}\left(\vec{x}^{\prime}, t^{\prime}\right)=\Phi(\vec{x}, t)-\ddot{\vec{\xi}} \cdot \vec{x}, \quad \nabla^{\prime} \Phi^{\prime}=\nabla \Phi-\ddot{\vec{\xi}} .
$$

Hence the splitting of the left-hand-side of (B.7) into an inertial term $m \ddot{\vec{x}}$ and a gravitational term $m \nabla \Phi$ has no objective significance as this splitting is frame-dependent: gravitational fields cannot be locally separated from translational-acceleration fields.

Integrable flat connection associated with the Galilean transformations does not remain invariant under Newtonian transformations (B.8). Indeed, using the transformation law of connection coefficients (see, for example, [63]. Einstein summation convention is assumed)

$$
\Gamma_{b c}^{\prime a}(x)=\frac{\partial x^{m}}{\partial x^{\prime b}} \frac{\partial x^{n}}{\partial x^{\prime c}} \frac{\partial x^{\prime a}}{\partial x^{l}} \Gamma_{m n}^{l}(x)+\frac{\partial x^{\prime a}}{\partial x^{l}} \frac{\partial^{2} x^{l}}{\partial x^{\prime b} \partial x^{\prime c}},
$$

one finds that if the connection coefficients are zero in some Newtonian frame, they don't remain zero after transformation (B.8). Namely, (B.11) shows that the nonzero connection coefficients in Newtonian coordinates are $\Gamma_{00}^{i}$, $i=1,2,3$, and under (B.8) they transform according to

$$
\Gamma_{00}^{\prime i}=\Gamma_{00}^{i}-\ddot{\xi}^{i} .
$$


Comparing with (B.9), we see that the connection covariant under Newtonian transformations $(\underline{B .8})$ and $(\underline{B} .10)$ is

$$
\Gamma_{00}^{i}=\frac{\partial \Phi}{\partial x^{i}} \equiv \Phi_{, i} \text { if } i=1,2,3, \quad \Gamma_{b c}^{a}=0 \text { otherwise. }
$$

For such connection, the free fall equation $\ddot{\vec{x}}+\nabla \Phi=0$ takes the form of geodesic equation

$$
\frac{d^{2} x^{a}}{d t^{2}}+\Gamma_{b c}^{a} \frac{d x^{b}}{d t} \frac{d x^{c}}{d t}=0 .
$$

These geodesics are a generalization of the notion of a "straight line" in the presence of gravity. As Edmund Whittaker once remarked "gravitation simply represents a continual effort of the universe to straighten itself out" [64]. More formally, gravity is a non-integrable, symmetric connection, whose geodesics are the free fall trajectories [58]. However, to make this last statement accurate, one must also geometrize the Poisson equation, which in the usual formulation of Newtonian gravity determines the gravitational potential. This can be done in the following way [64, 65].

In Newtonian spacetime, we have two degenerate metrics: temporal metric $t_{a b}$ and flat spatial metric $h^{a b}$. They can be motivated as follows [64]. In Minkowski spacetime, and for coordinates $(t, x, y, z)$, we have covariant metric tensor $g_{a b}=\operatorname{diag}\left(1,-1 / c^{2},-1 / c^{2},-1 / c^{2}\right)$ and its inverse contravariant tensor $g^{a b}=\operatorname{diag}\left(1,-c^{2},-c^{2},-c^{2}\right)$. When the light velocity $c$ goes to infinity, well defined limits $t_{a b}=\lim _{c \rightarrow \infty} g_{a b}$ and $h^{a b}=\lim _{c \rightarrow \infty}\left(\frac{-g^{a b}}{c^{2}}\right)$ will give, correspondingly, Newtonian temporal and spatial metrics. These metrics are assumed to obey orthogonality and compatibility conditions

$$
t_{a b} h^{a b}=0, \quad \nabla_{a} t_{b c}=0, \quad \nabla_{a} h^{b c}=0 .
$$

The first equation can clearly be motivated by $c \rightarrow \infty$ limit of the relation $g_{a b}\left(g^{a b} / c^{2}\right)=4 / c^{2}$. Note that the compatibility conditions don't uniquely determine the connection, in contrast to general relativity where $\nabla_{\mu} g_{\nu \lambda}=0$ metric-compatibility condition uniquely determines the connection. In this sense, the spacetime of geometrized Newtonian gravity is not metric (the connection coefficients are not expressible in terms of a metric tensor), but only affine [66].

From connection coefficients (B.13) , the Riemann tensor can be calculated with the result

$$
R_{0 j 0}^{i}=\partial_{j} \Gamma_{00}^{i}=\frac{\partial^{2} \Phi}{\partial x^{i} \partial x^{j}}, \quad R_{b c d}^{a}=0 \text { otherwise. }
$$


Relations (B.13) and (B.16) can be expressed in covariant form:

$$
\Gamma_{b c}^{a}=t_{b c} h^{a d} \nabla_{d} \Phi, \quad R_{b c d}^{a}=t_{b d} h^{a e} \nabla_{e} \nabla_{c} \Phi .
$$

Then $R_{a b} \equiv R_{a c b}^{c}=t_{a b} h^{c e} \nabla_{e} \nabla_{c} \Phi=t_{a b} \nabla^{2} \Phi$ and the Poisson equation $\nabla^{2} \Phi=$ $4 \pi G \rho$ takes the covariant form

$$
R_{a b}=4 \pi G \rho t_{a b} .
$$

Note that the gravitational potential $\Phi$ completely disappeared in (B.14) and (B.18) and everything is expressed exclusively through purely geometric quantities. Newtonian gravity, like the general theory of relativity, can also be considered as a spacetime geometry. At that this geometry becomes dynamical: "spacetime tells matter how to move; matter tells spacetime how to curve" [67].

The purpose of this rather long section was to convince the reader that free fall plays a central role in the geometrization of gravity not only in the general theory of relativity, but already in Newtonian theory. In fact, free fall defines a projective structure in spacetime and, as such, is necessary for understanding the foundations of any reasonable theory of spacetime and gravity [68]. Then an interesting question arises: what does the weak equivalence principle mean in quantum theory?

Of course, the understanding that it was impossible to locally distinguish between gravity and acceleration was Einstein's insight, who grandly call it the "most fortunate thought in my life" [69].

\section{Appendix C. Accelerated reference frame and gravity in classical mechanics}

We begin this section with a reminder of the meaning of passive and active transformations (see, for example, [70]). Passive transformation means one goes from one reference frame to another which is displaced, rotated, etc. relative to the original one. The system itself remains unchanged. Under active transformation it is the system under study which is displaced, rotated, etc. The observer's reference frame remains unchanged. Therefore, an active transformation is simply a change of coordinates in the same reference frame.

An example of a passive transformation is the transition from an inertial reference frame to an accelerated reference frame. Imagine two reference frames $S$ and $S^{\prime}$ whose origins coincide at $t=0$. Suppose $S$ is an inertial 
reference frame, while $S^{\prime}$ experiences a free fall in the gravitational field $\vec{g}$ present in the frame $S$. It is clear that coordinates of some event in frames $S$ and $S^{\prime}$ are related by relation (B.8) with $\ddot{\vec{\xi}}=\vec{g}$. In particular, if $\vec{g}$ points downwards, opposite to the $x$-axis, and the free fall begins at $t=0$ with zero relative velocity, we will have

$$
x^{\prime}=x+\frac{1}{2} g t^{2}, \quad y^{\prime}=y, \quad z^{\prime}=z, \quad t^{\prime}=t .
$$

This is the passive transformation we will use throughout this paper.

Note that a solution of the Euler-Lagrange equation of motion, which expresses the final coordinates $x_{f}$ through the initial coordinates $x_{i}$, can be considered as an active transformation since both coordinates are calculated relative to the same reference frame.

In reference frame $S$, the gravitational potential is $\Phi=g x$. Then, according to (B.13), the only non-zero connection coefficient is $\Gamma_{00}^{x}=g$, and (B.12) shows that in the frame $S^{\prime}$ all connection coefficients vanish. Therefore, gravity is absent in this frame in conformity with Einstein's principle of equivalence.

In the frame $S$, the equation of motion follows from the Lagrangian $\mathcal{L}_{g}=$ $\frac{m \dot{x}^{2}}{2}-m g x$, while in the frame $S^{\prime}$ the motion is free and the corresponding Lagrangian is equal to $\mathcal{L}_{f}=\frac{m \dot{x}^{\prime 2}}{2}$. These two Lagrangians are equivalent, as they should be. Indeed, expressing $x$ through $x^{\prime}$ from (C.1), we get

$$
\mathcal{L}_{g}=\frac{1}{2} m \dot{x}^{\prime 2}+m g^{2} t^{2}-m g\left(x^{\prime}+\dot{x}^{\prime} t\right)=\mathcal{L}_{f}+\frac{d \Lambda}{d t}, \quad \Lambda=\frac{1}{3} m g^{2} t^{3}-m g x^{\prime} t .
$$

However, any two Lagrangians that differ by a total time derivative are equivalent in the sense that they give the same equations of motion [70]. This equivalence is a mathematical expression of Einstein's principle of equivalence in classical mechanics.

As it is well known, in the quasiclassical approximation the phase of the quantum wave function coincides with the classical action integral along the classical trajectory [71, 72]. Therefore, equation (C.2) suggest that wave functions of a quantum particle in the uniform gravitational field and a free quantum particle in the freely falling non-inertial reference frame differ only in phase and this phase difference equals to $\Lambda / \hbar$.

The very same conclusion can be reached from the Hamiltonian perspective. If $\mathcal{L}_{g}$ is expressed through $x^{\prime}$, as in (C.2), then the corresponding 
canonical momentum is $p^{\prime}=\frac{\partial \mathcal{L}_{g}}{\partial \dot{x}^{\prime}}=m \dot{x}^{\prime}-m g t$. But C.1 indicates that $\dot{x}^{\prime}-g t=\dot{x}$, and therefore $p^{\prime}=p$. As we see, transition to the freely falling frame is equivalent to the following canonical transformation

$$
x^{\prime}=x+\frac{1}{2} g t^{2}, \quad p^{\prime}=p .
$$

This transformation is time dependent and thus the new Hamiltonian is [70]

$$
\mathcal{H}^{\prime}\left(x^{\prime}, p^{\prime}, t\right)=\mathcal{H}\left(x\left(x^{\prime}, p^{\prime}\right), p\left(x^{\prime}, p^{\prime}\right), t\right)+\frac{\partial F\left(x\left(x^{\prime}, p^{\prime}\right), p^{\prime}, t\right)}{\partial t},
$$

where $F\left(x, p^{\prime}, t\right)$ is the second type Legendre generator of the canonical transformation (C.3) [70]:

$$
p=\frac{\partial F\left(x, p^{\prime}, t\right)}{\partial x}, \quad x^{\prime}=\frac{\partial F\left(x, p^{\prime}, t\right)}{\partial p^{\prime}} .
$$

If we put $x^{\prime}=x+g t^{2} / 2$ in the second equation of (C.5) and integrate, we get $F\left(x, p^{\prime}, t\right)=p^{\prime}\left(x+\frac{1}{2} g t^{2}\right)+G(x, t)$, and then the first equation will give $p^{\prime}=p=\frac{\partial F\left(x, p^{\prime}, t\right)}{\partial x}=p^{\prime}+\frac{\partial G(x, t)}{\partial x}$. Therefore, $G(x, t)=f(t)$, where $f(t)$ is an arbitrary function of time, and the Legendre generator finally takes the form

$$
F\left(x, p^{\prime}, t\right)=p^{\prime}\left(x+\frac{1}{2} g t^{2}\right)+f(t) .
$$

In light of $p^{\prime}=p=m \dot{x}=m\left(\dot{x}^{\prime}-g t\right)$, we get from (C.4)

$$
\begin{aligned}
& \mathcal{H}^{\prime}= \frac{p^{\prime 2}}{2 m}+m g\left(x^{\prime}-\frac{1}{2} g t^{2}\right)+m g t\left(\dot{x}^{\prime}-g t\right)+\dot{f}(t)= \\
& \frac{p^{\prime 2}}{2 m}+\frac{d}{d t}\left(m g x^{\prime} t\right)-\frac{3}{2} m g^{2} t^{2}+\dot{f}(t) .
\end{aligned}
$$

If now we choose $f(t)=\frac{1}{6} m g^{2} t^{3}$, we get

$$
\mathcal{H}^{\prime}=\frac{p^{2}}{2 m}-\frac{d \Lambda}{d t}
$$

This relation shows that in the freely falling reference frame $S^{\prime}$, the Hamiltonian $\mathcal{H}^{\prime}$ of a particle in a homogeneous gravitational field differs from the free Hamiltonian $\frac{p^{2}}{2 m}$ by a gauge term $-\frac{d \Lambda}{d t}$. Therefore, again it is expected that the corresponding wave functions in frames $S$ and $S^{\prime}$ differ only by a phase $\Lambda / \hbar$. As we have seen in the main text, this is indeed true in quantum mechanics. 


\section{Appendix D. KvN mechanics: an introductory review}

The one-dimensional Schrödinger equation, which describes the dynamics of a quantum particle in terms of the wave function $\psi(x, t)$, is given by

$$
i \hbar \frac{\partial \psi(x, t)}{\partial t}=-\frac{\hbar^{2}}{2 m} \frac{\partial^{2} \psi(x, t)}{\partial x^{2}}+V(x) \psi(x, t) .
$$

The probability density to find a quantum particle at a particular position $x$ at a time $t$ is given by modulus squared of the wave function $\rho(x, t)=$ $|\psi(x, t)|^{2}$. Interestingly, there is a close relationship of (D.1) with the diffusion equation [73]. For a free particle $(V=0)$, if we define the imaginary time $\tau=i t$, then the Schrödinger equation will look like a diffusion equation (also called the heat equation) with a diffusion coefficient $D=\frac{\hbar}{2 m} 2$ :

$$
\frac{\partial \psi}{\partial \tau}=D \frac{\partial^{2} \psi}{\partial x^{2}}
$$

Let's make the Hopf-Cole transform [75]

$$
\psi(x, t)=e^{-\frac{1}{2 D} \int_{0}^{x} u(y, t) d y}
$$

in (D.2). Then we get

$$
\frac{\partial}{\partial t}\left(\int_{0}^{x} u(y, t) d y\right)+\frac{1}{2} u^{2}=D \frac{\partial u}{\partial x}
$$

and taking the partial derivative with respect to $x$, we get the Burgers equation for $u(x, t)$ :

$$
\frac{\partial u}{\partial t}+u \frac{\partial u}{\partial x}=D \frac{\partial^{2} u}{\partial x^{2}}
$$

The Burgers equation is an approximation of the Navier-Stokes equations when pressure is neglected, but the influence of the nonlinear and viscous terms is preserved [76]. In light of similarities between (D.1) and (D.2), it is expected that the Schrödinger equation can also have a hydrodynamic

\footnotetext{
${ }^{2}$ In a remarkable paper 74] Nelson proposed a derivation of the Schrödinger equation, suggesting that a quantum particle is subject to Brownian motion with the diffusion coefficient $D=\frac{\hbar}{2 m}$.
} 
formulation. In a seminal paper [77] Madelung showed that this is indeed true.

Madelung transform is a complex analog of the Hopf-Cole transform (D.3):

$$
\psi(x, t)=e^{\frac{1}{2} \ln \rho(x, t)+\frac{i}{\hbar} S(x, t)}=\sqrt{\rho(x, t)} e^{\frac{i}{\hbar} S(x, t)} .
$$

If we substitute (D.5) into the Schrödinger equation (D.1), we obtain the following equations after separation of the real and imaginary parts:

$$
\begin{aligned}
& \frac{\partial \rho}{\partial t}+\frac{\partial}{\partial x}(\rho v)=0 \\
& \frac{\partial S}{\partial t}+\frac{1}{2 m}\left(\frac{\partial S}{\partial x}\right)^{2}+V(x, t)+Q(x, t)=0
\end{aligned}
$$

where

$$
Q(x, t)=-\frac{\hbar^{2}}{2 m \sqrt{\rho}}\left(\frac{\partial^{2} \sqrt{\rho}}{\partial x^{2}}\right), \quad v=\frac{1}{m} \frac{\partial S}{\partial x} .
$$

In the three-dimensional case, the corresponding equations are [77]

$$
\begin{aligned}
& \frac{\partial \rho}{\partial t}+\nabla \cdot(\rho \vec{v})=0 \\
& \frac{\partial S}{\partial t}+\frac{1}{2 m}(\nabla S)^{2}+V(x, t)+Q(x, t)=0
\end{aligned}
$$

and

$$
Q=-\frac{\hbar^{2}}{2 m \sqrt{\rho}} \nabla^{2} \sqrt{\rho}=-\frac{\hbar^{2}}{2 m}\left[(\nabla \ln \sqrt{\rho})^{2}+\nabla^{2} \ln \sqrt{\rho}\right], \quad v=\frac{1}{m} \nabla S .
$$

Using $\epsilon_{i j k} \epsilon_{k m n}=\delta_{i m} \delta_{j n}-\delta_{i n} \delta_{j m}$, it is easy to prove that $\vec{v} \times(\nabla \times \vec{v})=$ $\frac{1}{2} \nabla v^{2}-(\vec{v} \cdot \nabla) \vec{v}$. But $\nabla \times \vec{v}=0$ since $\vec{v}$ is a gradient. Therefore, if we apply $\frac{1}{m} \nabla$ operator to both sides of the second equation of (D.8), we get

$$
\frac{\partial \vec{v}}{\partial t}+(\vec{v} \cdot \nabla) \vec{v}=-\frac{1}{m} \nabla(V+Q) .
$$

This last equation has close analogy with an irrotational flow of a perfect fluid of density $\rho$ in a gravitational field $\vec{g}$ described by Euler equation [78]

$$
\frac{\partial \vec{v}}{\partial t}+(\vec{v} \cdot \nabla) \vec{v}=\vec{g}-\frac{\nabla p}{\rho}
$$


where $p$ is the pressure.

Indeed, using $\nabla_{j} \ln \sqrt{\rho}=\nabla_{j} \rho /(2 \rho)$, we get

$$
\begin{aligned}
& \left(-\frac{2 m}{\hbar^{2}}\right) \nabla_{i} Q=\left[2\left(\nabla_{j} \ln \sqrt{\rho}\right) \nabla_{i} \nabla_{j} \ln \sqrt{\rho}+\nabla_{i} \nabla_{j} \nabla_{j} \ln \sqrt{\rho}\right]= \\
& \frac{1}{\rho}\left[\left(\nabla_{j} \rho\right) \nabla_{i} \nabla_{j} \ln \sqrt{\rho}+\rho \nabla_{j} \nabla_{i} \nabla_{j} \ln \sqrt{\rho}\right]=\frac{1}{\rho} \nabla_{j}\left(\rho \nabla_{i} \nabla_{j} \ln \sqrt{\rho}(x \mathrm{D} .12)\right.
\end{aligned}
$$

Therefore,

$$
\frac{1}{m} \nabla_{i} Q=\frac{1}{m \rho} \nabla_{j} \sigma_{i j}, \quad \sigma_{i j}=-\frac{\hbar^{2}}{2 m} \rho \nabla_{i} \nabla_{j} \ln \sqrt{\rho} .
$$

As a result, comparing (D.10) and (D.11), we see that the Madelung equations (D.8) can be considered as a description of the hydrodynamics of a specific fluid with mass density $m \rho$, in which the pressure tensor $p \delta_{i j}$ is replaced by the tensor $\sigma_{i j}[79,80]$.

Madelung representation of the Schrödinger equation proved to be useful and is extensively used in the description of physical phenomena like superfluidity, Bose-Einstein condensation, quantum plasmas, to name a few. Oddly enough, Madelung's contemporaries paid very little attention to this approach, and Pauli even expressed the opinion that it was not very interesting [81].

It is noteworthy that a fundamental equation of statistical mechanics, the Liouville equation

$$
\frac{\partial f(\vec{x}, \vec{p}, t)}{\partial t}=\{H(\vec{x}, \vec{p}, t), f(\vec{x}, \vec{p}, t)\}, \quad\{H, f\}=\frac{\partial H}{\partial x_{i}} \frac{\partial f}{\partial p_{i}}-\frac{\partial H}{\partial p_{i}} \frac{\partial f}{\partial x_{i}},
$$

also admits a hydrodynamic interpretation. This can be shown as follows [82].

Let's make hydrodynamic substitution $f(\vec{x}, \vec{p}, t)=\rho(\vec{x}, t) \delta(\vec{p}-m \vec{v}(\vec{x}, t))$ in the Liouville equation and integrate over $\vec{p}$ under the assumption of the usual Hamiltonian $H=\vec{p}^{2} /(2 m)+V(\vec{x})$. Since

$$
\int \frac{\partial V}{\partial x_{i}} \frac{\partial f}{\partial p_{i}} d \vec{p}=\rho \frac{\partial V}{\partial x_{i}} \int \frac{\partial}{\partial p_{i}} \delta(\vec{p}-m \vec{v}) d \vec{p}=0,
$$

and

$$
\int \frac{p_{i}}{m} \frac{\partial f}{\partial x_{i}} d \vec{p}=\frac{\partial}{\partial x_{i}}\left(\rho \int \frac{p_{i}}{m} \delta(\vec{p}-m \vec{v}) d \vec{p}\right)=\frac{\partial}{\partial x_{i}}\left(\rho v_{i}\right),
$$


we immediately obtain the continuity equation (the first equation of the Euler system (D.8)).

To obtain Euler's momentum equation, we multiply the Liouville equation by $p_{i}$ and integrate over $\vec{p}$. Since

$$
\int \frac{\partial V}{\partial x_{j}} \frac{\partial f}{\partial p_{j}} p_{i} d \vec{p}=\rho \frac{\partial V}{\partial x_{j}} \int p_{i} \frac{\partial}{\partial p_{j}} \delta(\vec{p}-m \vec{v}) d \vec{p}=-\rho \frac{\partial V}{\partial x_{i}}
$$

and

$$
\int \frac{p_{j}}{m} \frac{\partial f}{\partial x_{j}} p_{i} d \vec{p}=\frac{\partial}{\partial x_{j}}\left(\rho \int \frac{p_{j}}{m} p_{i} \delta(\vec{p}-m \vec{v}) d \vec{p}\right)=m \frac{\partial}{\partial x_{j}}\left(\rho v_{i} v_{j}\right),
$$

we obtain

$$
\frac{\partial}{\partial t}\left(\rho v_{i}\right)+\frac{\partial}{\partial x_{j}}\left(\rho v_{j} v_{i}\right)=-\frac{\rho}{m} \frac{\partial V}{\partial x_{i}} .
$$

Because of the continuity equation, (D.15) is equivalent to (D.10) without the quantum potential $Q$. Indeed, the left hand side of (D.15) equals to

$$
\rho \frac{\partial v_{i}}{\partial t}+v_{i}\left(\frac{\partial \rho}{\partial t}+\frac{\partial}{\partial x_{j}}\left(\rho v_{j}\right)\right)+\rho v_{j} \frac{\partial v_{i}}{\partial x_{j}}=\rho \frac{\partial v_{i}}{\partial t}+\rho v_{j} \frac{\partial v_{i}}{\partial x_{j}} .
$$

Since both the Schrödinger equation and the Liouville equation admit a hydrodynamic interpretation, the natural question is whether it is possible to formulate classical mechanics in terms of a wave function $\psi(x, p, t)$ in phase space. This wave function must be of Schrödinger type, that is, the following postulates usually attributed to quantum theory should be satisfied:

- The states of a classical mechanical system are represented by normalized vectors $|\psi\rangle$ of a complex Hilbert space;

- Observables are given by self-adjoint operators acting on this Hilbert space;

- The expectation value of an observable $\hat{A}$ in a given state $|\psi\rangle$ is $\bar{A}=$ $\langle\psi|\hat{A}| \psi\rangle$, where the inner product equals to

$$
\langle\psi \mid \phi\rangle=\int d q d p \psi^{*}(p, q, t) \phi(p, q, t),
$$

- If $|A\rangle$ is an eigenvector of the observable $\hat{A}$ with eigenvalue $A$, the probability that a measurement of the observer $\hat{A}$ in a given state $|\psi\rangle$ yields $A$ is $|\langle A \mid \psi\rangle|^{2}$. 
Such kind of scenario can be realized as follows [83, 84]. The unitary operators $\hat{U}(t)$ that connect state vectors at time 0 to state vectors at time $t$,

$$
|\psi(t)\rangle=\hat{U}(t)|\psi(0)\rangle
$$

constitute a unitary representation of the one-parameter group of time translations. According to Stone's theorem, $\hat{U}(t)=e^{-\frac{i}{\hbar} \hat{H}}$, where $\hat{H}$ is Hermitian (a Physicist's proof of Stone's theorem can be found in [85], and some refinements in [86]). Then (D.17) imply

$$
i \hbar \frac{d}{d t}|\psi(t)\rangle=\hat{H}|\psi(t)\rangle
$$

As a fundamental principle determining $\hat{H}$, we assume that the evolution of expectation values of the coordinate and momentum operators is governed by the Ehrenfest theorem (a kind of correspondence principle that requires Newton's equations for these expectation values)

$$
\frac{d}{d t}\langle\hat{x}\rangle=\left\langle\frac{\hat{p}}{m}\right\rangle, \quad \frac{d}{d t}\langle\hat{p}\rangle=\left\langle-\hat{V}^{\prime}(\hat{x})\right\rangle,
$$

while the operators $\hat{x}$ and $\hat{p}$ themselves commute:

$$
[\hat{x}, \hat{p}]=0 .
$$

Note that in order to obtain the operator $\hat{V}^{\prime}(\hat{x})$ we need to take the derivative of the potential $V(x)$ by treating $x$ as a $c$-number and then converting $x \rightarrow \hat{x}$ in the result [84].

Because of (D.18) and (D.19),

$$
i \hbar \frac{d}{d t}\langle\hat{x}\rangle=i \hbar\langle\psi(t)|\hat{x}| \psi(t)\rangle=\langle\psi(t)|[\hat{x}, \hat{H}]| \psi(t)\rangle=i \hbar\left\langle\psi(t)\left|\frac{\hat{p}}{m}\right| \psi(t)\right\rangle,
$$

and similarly for $i \hbar \frac{d}{d t}\langle\hat{p}\rangle$. As relations (D.19) must be satisfied for any state vector $|\psi(t)\rangle$, we get

$$
\frac{i}{\hbar}[\hat{H}, \hat{x}]=\frac{\hat{p}}{m}, \quad \frac{i}{\hbar}[\hat{H}, \hat{p}]=-\hat{V}^{\prime}(\hat{x}) .
$$

However, in light of (D.20) , if $\hat{H}$ is a function of only $\hat{x}$ and $\hat{p}$, the commutation relations $(\mathrm{D} .21)$ are clearly impossible. Therefore we introduce two new operators $\hat{X}$ and $\hat{P}$ with commutation relations

$$
[\hat{X}, \hat{p}]=i \hbar,[\hat{X}, \hat{P}]=0,[\hat{p}, \hat{P}]=0,[\hat{x}, \hat{X}]=0,[\hat{x}, \hat{p}]=0,[\hat{x}, \hat{P}]=i \hbar
$$


and suppose that $\hat{H}(\hat{x}, \hat{X}, \hat{p}, \hat{P})$ depends on these new operators too. Then

$$
\frac{i}{\hbar}[\hat{H}, \hat{x}]=\frac{\partial \hat{H}}{\partial \hat{P}}, \quad \frac{i}{\hbar}[\hat{H}, \hat{p}]=-\frac{\partial \hat{H}}{\partial \hat{X}},
$$

and comparing with (D.21) we see that

$$
\hat{H}=\frac{\hat{p} \hat{P}}{m}+\hat{V}^{\prime}(\hat{x}) \hat{X}+f(\hat{x}, \hat{p}, t)
$$

where $f(\hat{x}, \hat{p}, t)$ is an arbitrary real function.

Since $\hat{x}$ and $\hat{p}$ are commuting operators, we can use a basis of their common eigenstates $|x, p\rangle$ to define the wave function $\psi(x, p, t)=\langle x, p \mid \psi(t)\rangle$. In this $(x, p)$-representation

$$
\hat{P}=-i \hbar \frac{\partial}{\partial x}, \quad \hat{X}=i \hbar \frac{\partial}{\partial p}
$$

and the Schrödinger equation (D.18) takes the form

$$
\frac{\partial \psi(x, p, t)}{\partial t}=\left(V^{\prime}(x) \frac{\partial}{\partial p}-\frac{p}{m} \frac{\partial}{\partial x}-\frac{i}{\hbar} f(x, p, t)\right) \psi(x, p, t) .
$$

Then the probability density $\rho(x, p, t)=\psi^{*}(x, p, t) \psi(x, p, t)$ satisfies the equation

$$
\frac{\partial \rho(x, p, t)}{\partial t}=V^{\prime}(x) \frac{\partial \rho(x, p, t)}{\partial p}-\frac{p}{m} \frac{\partial \rho(x, p, t)}{\partial x}=\left\{H_{c l}, \rho\right\}
$$

which is nothing but the Liouville equation (D.14) with the classical Hamiltonian $H_{c l}=\frac{p^{2}}{2 m}+V(x)$.

As we see, the presence of the arbitrary function $f(x, p, t)$ in (D.24) does not affect the observed probability density $\rho$ and, thus, it can be safely ignored. In fact, this gauge freedom in the choice of the Hamiltonian (D.24) (the Liouville operator) is related to the invariance of the KvN probability density function under the phase transformation of the corresponding wave function [87] and reflects the fact that the phase of the wave function is irrelevant in classical theory.

If $f(x, p, t)=0$, then both $\rho(x, p, t)$ and $\psi(x, p, t)$ satisfy the same Liouville equation (D.27). This difference between KvN mechanics and quantum 
mechanics is due to the fact that in the $(x, p)$-representation the Liouville equation is linear in derivatives.

Interesting perspective on KvN mechanics was given by Sudarshan [88, 89]. We can consider $(x, X, p, P)$ as the phase space of a quantum system with twice as many degrees of freedom than the classical system $(x, p)$. In the subspace $(x, p)$, the classical dynamics arises due to the very unusual form of the quantum Hamiltonian (D.24), which is linear in the classically hidden variables $X$ and $P$.

The dynamics of a $\mathrm{KvN}$ particle will look more quantum-like in the $(x, X)$ representation. In this representation $\hat{x}$ and $\hat{X}$ operators are diagonal, while

$$
\hat{P}=-i \hbar \frac{\partial}{\partial x}, \quad \hat{p}=-i \hbar \frac{\partial}{\partial X},
$$

and the Schrödinger equation (D.18) takes the form

$$
i \hbar \frac{\partial \psi(x, X, t)}{\partial t}=-\frac{\hbar^{2}}{m} \frac{\partial^{2} \psi(x, X, t)}{\partial x \partial X}+X \frac{d V}{d x} \psi(x, X, t) .
$$

Hilbert space approach to classical mechanics was pioneered by Koopman [13] and von Neumann [14]. A detailed up-to-date pedagogical review is given in [90], where the interested reader can also find relevant references regarding contemporary research related to $\mathrm{KvN}$ mechanics (in this regard, see also [87]).

\section{Acknowledgments}

We are grateful to the anonymous referee for comments that helped to improve the presentation of the manuscript. The work of Z.K.S. is supported by the Ministry of Education and Science of the Russian Federation.

\section{References}

[1] R. H. Dicke, Republication of: The theoretical significance of experimental relativity, Gen. Relativ. Gravit. 51 (2019), 57 (2019). DOI:10.1007/s10714-019-2509-2

[2] C. M. Will, Theory and Experiment in Gravitational Physics (Cambridge: Cambridge University Press, 1993).

DOI:10.1017/9781316338612 
[3] A. M. Nobili and A. Anselmi, Relevance of the weak equivalence principle and experiments to test it: lessons from the past and improvements expected in space, Phys. Lett. A 382 (2018), 2205-2218. DOI:10.1016/j.physleta.2017.09.027

[4] C. M. Will, The Confrontation between General Relativity and Experiment, Living Rev. Rel. 17 )2014), 4. DOI:10.12942/lrr-2014-4

[5] J. Ehlers, The Nature and Structure of Spacetime, in J. Mehra (Ed.), The Physicist's Conception of Nature (Dordrecht: Reidel, 1973), pp. 71-91.

[6] E. Di Casola, S. Liberati and S. Sonego, Nonequivalence of equivalence principles, Am. J. Phys. 83 (2015), 39-46. DOI:10.1119/1.4895342

[7] G. Muñoz and P. Jones, The equivalence principle, uniformly accelerated reference frames, and the uniform gravitational field, Am. J. Phys. 78 (2010), 377-383. DOI:10.1119/1.3272719

[8] J. Norton, What was Einstein's principle of equivalence? Stud. Hist. Phil. Sci. A 16 (1985), 203-246. DOI:10.1016/0039-3681(85)90002-0

[9] D. Greenberger, The role of equivalence in Quantum Mechanics, Annals Phys. 47 (1968), 116-126. DOI:10.1016/0003-4916(68)90229-7

[10] H. Padmanabhan and T. Padmanabhan, Non-relativistic limit of quantum field theory in inertial and non-inertial frames and the Principle of Equivalence, Phys. Rev. D 84 (2011), 085018; Addendum: Phys. Rev. D 90 (2014), 089908.

[11] E. Okon and C. Callender, Does Quantum Mechanics Clash with the Equivalence Principle — and Does it Matter?, Eur. J. Phil. Sci. 1 (2001), 133-145. DOI:10.1007/s13194-010-0009-z

[12] G. Rosen, Galilean Invariance and the General Covariance of Nonrelativistic Laws, Am. J. Phys. 40 (1972), 683-687. DOI:10.1119/1.1986618

[13] B. O. Koopman, Hamiltonian Systems and Transformations in Hilbert Space, Proc. Nat. Acad. Sci. 17 (1931), 315-318.

DOI:10.1073/pnas.17.5.315 
[14] J. von Neumann, Zur Operatorenmethode In Der Klassischen Mechanik, Annals Math. 33 (1932), 587-642; DOI:10.2307/1968537 Zusatze Zur Arbeit "Zur Operatorenmethode...", Annals Math. 33 (1932), 789-791. DOI:10.2307/1968225

[15] R. P. Feynman, An Operator calculus having applications in quantum electrodynamics, Phys. Rev. 84 (1951), 108-128.

DOI:10.1103/PhysRev.84.108

[16] V. S. Popov, Feynman disentangling of noncommuting operators and group representation theory, Phys. Usp. 50 (2207), 1217-1238.

DOI:10.1070/PU2007v050n12ABEH006401

[17] F. Soto-Eguibar and H. M. Moya-Cessa, Solution of the Schrödinger equation for a Linear potential using the extended Baker-CampbellHausdorff formula, Appl. Math. Inf. Sci. 9 (2015), 175-181.

DOI:10.12785/amis/090123

[18] M. Nauenberg, Einstein's equivalence principle in quantum mechanics revisited, Am. J. Phys. 84 (2016), 879-882. DOI:10.1119/1.4962981

[19] D. M. Greenberger and A. W. Overhauser, Coherence effects in neutron diffraction and gravity experiments, Rev. Mod. Phys. 51 (1979), 43-78. DOI:10.1103/RevModPhys.51.43

[20] R. Colella, A. W. Overhauser and S. A. Werner, Observation of gravitationally induced quantum interference, Phys. Rev. Lett. 34 (1975), 1472-1474. DOI:10.1103/PhysRevLett.34.1472

[21] R. Colella and A. W. Overhauser, How the COW happened, Physica B 385-386 (2006), 1408-1410. DOI:10.1016/j.physb.2006.05.200

[22] C. Anastopoulos and B. L. Hu, Equivalence principle for quantum systems: dephasing and phase shift of free-falling particles, Class. Quantum Grav. 35 (2018), 035011. DOI:10.1088/1361-6382/aaa0e8

[23] J. J. Sakurai, Modern Quantum Mechanics (Addison-Wesley: Reading, 1994), p. 129.

[24] K. Varjú and L. H. Ryder, General relativistic treatment of the ColellaOverhauser-Werner experiment on neutron interference in a gravitational field, Am. J. Phys. 68 (2000), 404-409. DOI: 10.1119/1.19454 
[25] P. D. Mannheim, The equivalence principle in classical mechanics and quantum mechanics, in V. Dvoeglazov (Ed.) Photon: Old problems in Light of New Ideas (Nova Science: New York, 2000), pp. 190-206.

[26] P. D. Mannheim, Classical underpinnings of gravitationally induced quantum interference, Phys. Rev. A 57 (1998), 1260-1264.

DOI:10.1103/PhysRevA.57.1260

[27] B. Heacock, M. Arif, R. Haun, M. G. Huber, D. A. Pushin and A. R. Young, Neutron interferometer crystallographic imperfections and gravitationally induced quantum interference measurements, Phys. Rev. A 95 (2017), 013840. DOI:10.1103/PhysRevA.95.013840

[28] U. Bonse and T. Wroblewski, Measurement of Neutron Quantum Interference in Noninertial Frames, Phys. Rev. Lett. 51 (1983), 1401-1404. DOI:10.1103/physrevlett.51.1401

[29] L. Carroll, The Hunting of the Snark, an Agony in Eight Fits (Macmillan: London, 1876).

[30] L. A. Beauregard, Propagators in Nonrelativistic Quantum Mechanics, Am. J. Phys. 34 (1966), 324-332. DOI:10.1119/1.1972954

[31] B. R. Holstein, The linear potential propagator, Am. J. Phys. 65 (1997), 414-418. DOI:10.1119/1.18550

[32] F. A. Barone, H. Boschi-Filho and C. Farina, Three Methods for Computing the Feynman Propagator, Am. J. Phys. 71 (2003) 483-491. DOI:10.1119/1.1538573

[33] G. P. Arrighini, N. L. Durante and C. Guidotti, More on the quantum propagator of a particle in a linear potential, Am. J. Phys. 64 (1996), 1036-1041. DOI:10.1119/1.18323

[34] L. S. Brown and Y. Zhang, Path integral for the motion of a particle in a linear potential, Am. J. Phys. 62 (1994), 806-808. DOI:10.1119/1.17463

[35] N. Wheeler, Classical/Quantum Dynamics in a Uniform Gravitational Field: A. Unobstructed Free Fall, https://www.reed.edu/physics/faculty/wheeler/documents/Quantum\%20Mechanics/Mis Accessed 10 Aug 2020. 
[36] R. P. Kanwal, Generalized Functions: Theory and Applications (Birkhäuser: Boston, 2004).

[37] M. Wadati, The Free Fall of Quantum Particles, J. Phys. Soc. Japan 68 (1999), 2543-2546. DOI:10.1143/JPSJ.68.2543

[38] M. N. Berberan-Santos, E. N. Bodunov and L. Pogliani, Classical and quantum study of the motion of a particle in a gravitational field, J. Math. Chem. 37 (2005), 101-115. DOI:10.1007/s10910-004-1443-y

[39] L. Seveso and M. G. A. Paris, Can quantum probes satisfy the weak equivalence principle? Annals Phys. 380 (2017), 213-223.

DOI:10.1016/j.aop.2017.03.021

[40] B. R. Frieden, Physics from Fisher information: a unification (Cambridge University Press, Cambridge, 1998).

[41] N. Zettili, Quantum Mechanics: Concepts and Applications (Wiley: New York, 2009).

[42] D. Mauro, On Koopman-von Neumann waves, Int. J. Mod. Phys. A 17 (2002), 1301-1325. DOI:10.1142/S0217751X02009680

[43] A. S. Avanesov and V. I. Man'ko, Wave function of classical particlein linear potential, J. Russ. Laser Res. 34 (2013), 239-246.

DOI:10.1007/s10946-013-9347-6

[44] R. Geroch and P. S. Jang, Motion of a body in general relativity, J. Math. Phys. 16 (1975), 65-67. DOI:10.1063/1.522416

[45] L. Ryder, Introduction to General Relativity (Cambridge: Cambridge University Press, 2009). DOI:10.1017/CBO9780511809033

[46] B. M. Casper, Galileo and the fall of Aristotle: A case of historical injustice? Am. J. Phys. 45 (1977), 325-330. DOI:10.1119/1.10614

[47] C. G. Adler and B. L. Coulter, Galileo and the Tower of Pisa experiment, Am. J. Phys. 46 (1978), 199-201. DOI:10.1119/1.11165

[48] C. W. Groetsch, Aristotle's Fall, Am. Math. Monthly 105 (1998), 544547. DOI:10.2307/2589405 
[49] M. Segre, The Role of Experiment in Galileo's Physics, Arch. Hist. Ex. Sci. 23 (1980), 227-252. DOI:10.1007/BF00357045

[50] S. Straulino, Reconstruction of Galileo Galilei's experiment: the inclined plane, Phys. Educ. 43 (2008), 316-320. DOI:10.1088/00319120/43/3/012

[51] P. D. Sherman, Galileo and the Inclined Plane Controversy, Phys. Teacher 12 (1974), 343-348. DOI:10.1119/1.2350421

[52] S. Hacyan, Galileo and the equivalence principle: a faulty argument with the correct conclusion, Eur. J. Phys. 36 (2015), 065044. DOI:10.1088/0143-0807/36/6/065044

[53] J. G. Williams, S. G. Turyshev and D. Boggs, Lunar Laser Ranging Tests of the Equivalence Principle, Class. Quant. Grav. 29 (2012), 184004. DOI:10.1088/0264-9381/29/18/184004

[54] R. G. Keesing, The history of Newton's apple tree, Contemp. Phys. 39 (1998), 377-391. DOI:10.1080/001075198181874

[55] P. Fara, Newton: The Making of Genius (New York: Columbia University Press, 2003).

[56] D. S. Smith, Newton's Apple, Phys. Educ. 32 (1997), 129-131. DOI:10.1088/0031-9120/32/2/024

[57] R. L. Numbers and K. Kampourakis (Eds.), Newton's Apple and other Myths about Science (Cambridge: Harvard University Press, 2015).

[58] J. Ehlers, Survey of General Relativity Theory, in W. Israel (Ed.) Relativity, Astrophysics and Cosmology (Dordrecht: Reidel, 1973), pp. 1-125.

[59] A. Trautman, Comparison of Newtonian and Relativistic Theories of Space-Time, in B. Hoffmann (Ed.) Perspectives in Geometry and Relativity, Essays in honor of V. Hlavaty (Indiana University Press: Bloomington, 1966), pp. 413-425.

[60] P. Havas, Four-Dimensional Formulations of Newtonian Mechanics and Their Relation to the Special and the General Theory of Relativity, Rev. Mod. Phys. 36 (1964), 938-965. DOI:10.1103/RevModPhys.36.938 
[61] H. P. Künzle, Galilei and lorentz structures on space-time - comparison of the corresponding geometry and physics, Ann. Inst. Henri Poincaré, section A 17 (1972), 337-362.

[62] J. Bain, Theories of Newtonian gravity and empirical indistinguishability, Stud. Hist. Phil. Sci. B 35 (2004), 345-376.

DOI:10.1016/j.shpsb.2003.10.004

[63] C. J. Isham, Modern Differential Geometry for Physicists (World Scientific: Singapore, 2001).

[64] D. Malament, Gravity and Spatial Geometry, Stud. Logic Found. Math. 114 (1986), 405-411. DOI:10.1016/S0049-237X(09)70703-7

[65] D. B. Malament, Topics in the Foundations of General Relativity and Newtonian Gravitation Theory (University of Chicago Press: Chicago, 2012).

[66] P. Havas, Foundation Problems in General Relativity, in M. Bunge (Ed.), Delaware Seminar in the Foundations of Physics (SpringerVerlag: Berlin, 1967), pp. 124-148.

[67] J. A. Wheeler, A Journey into Gravity and Spacetime (Scientific American Library: New York, 1999).

[68] J. Ehlers, F. A. E. Pirani and A. Schild, Republication of: The geometry of free fall and light propagation, Gen. Relativ. Gravit. 44 (2012), 15871609. DOI:10.1007/s10714-012-1353-4

[69] W. Isaacson, Einstein: His Life and Universe (Simon \& Schuster: New York, 2008).

[70] M. G. Calkin, Lagrangian and hamiltonian mechanics (World Scientific: Singapore, 1998).

[71] P. A. M. Dirac, The Principles of Quantum Mechanics (Oxford University Press: Oxford, 1958).

[72] M. V. Berry and K. E. Mount, Semiclassical approximations in wave mechanics, Rept. Prog. Phys. 35 (1972), 315-397. DOI:10.1088/0034$4885 / 35 / 1 / 306$ 
[73] R. P. Feynman, R. B. Leighton and M. Sands, The Feynman Lectures on Physics, Vol. 3, Ch. 16 (Addison-Wesley: Reading, 1963).

[74] E. Nelson, Derivation of the Schrodinger equation from Newtonian mechanics, Phys. Rev. 150 (1966), 1079-1085.

DOI:10.1103/PhysRev.150.1079

[75] T.-P. Liu, Hopf-Cole transformation, Bull. Inst. Math. Acad. Sin. 12 (2017), 71-101. DOI:10.21915/BIMAS.2017103

[76] P. Orlandi, The Burgers equation, in P. Orlandi (Ed.), Fluid Flow Phenomena. Fluid Mechanics and Its Applications, vol 55 (Springer: Dordrecht, 2000). DOI:10.1007/978-94-011-4281-6_4

[77] E. Madelung, Quantentheorie in hydrodynamischer Form, Z. Phys. 40 (1927), 322-326. DOI:10.1007/BF01400372

[78] L. D. Landau and E. M. Lifshitz, Fluid Mechanics (Pergamon Press: London, 1987).

[79] T. Takabayasi, Remarks on the Formulation of Quantum Mechanics with Classical Pictures and on Relations between Linear Scalar Fields and Hydrodynamical Fields, Prog. Theor. Phys. 9 (1953), 187-222. DOI:10.1143/ptp/9.3.187

[80] P. R. Holland, The quantum theory of motion: an account of the de Broglie-Bohm causal interpretation of quantum mechanics (Cambridge University Press: Cambridge, 1993).

[81] E. A. Spiegel, Fluid dynamical form of the linear and nonlinear Schrödinger equations, Physica D 1 (1980), 236-240. DOI:10.1016/01672789(80)90015-9

[82] V. V. Vedenyapin and N. N. Fimin, The Liouville Equation, the Hydrodynamic Substitution, and the Hamilton-Jacobi Equation, Dokl. Math. 86 (2012), 697-699. DOI:10.1134/S1064562412050134

[83] D. I. Bondar, R. Cabrera, R. R. Lompay, M. Yu. Ivanov and H. A. Rabitz, Operational Dynamic Modeling Transcending Quantum and Classical Mechanics, Phys. Rev. Lett. 109 (2012), 190403.

DOI:10.1103/PhysRevLett.109.190403 
[84] F. Wilczek, Notes on Koopman von Neumann Mechanics, and A Step Beyond, http://frankwilczek.com/2015/koopmanVonNeumann02.pdf Accessed 10 Aug 2020.

[85] L. E. Ballentine, Quantum Mechanics: A Modern Development (World Scientific: Singapore, 1998), pp.65-66.

[86] V. S. Araujo, F. A. B. Coutinho and F. M. Toyama, The timedependent Schrödinger equation: the need for the Hamiltonian to be self-adjoint, Braz. J. Phys. 38 (2008), 178-187. DOI:10.1590/S010397332008000100030

[87] O. I. Chashchina, A. Sen and Z. K. Silagadze, On deformations of classical mechanics due to Planck-scale physics, Int. J. Mod. Phys. D (2020) https://doi.org/10.1142/S0218271820500704

[88] E. C. G. Sudarshan, Interaction between classical and quantum systems and the measurement of quantum observables, Pramana 6 (1976), 117126. DOI:10.1007/BF02847120

[89] T. N. Sherry and E. C. G. Sudarshan, Interaction Between Classical and Quantum Systems: A New Approach to Quantum Measurement, Phys. Rev. D 18 (1978), 4580-4589. DOI:10.1103/PhysRevD.20.857

[90] D. Mauro, Topics in Koopman-von Neumann Theory, PhD Thesis, arXiv:quant-ph/0301172. 